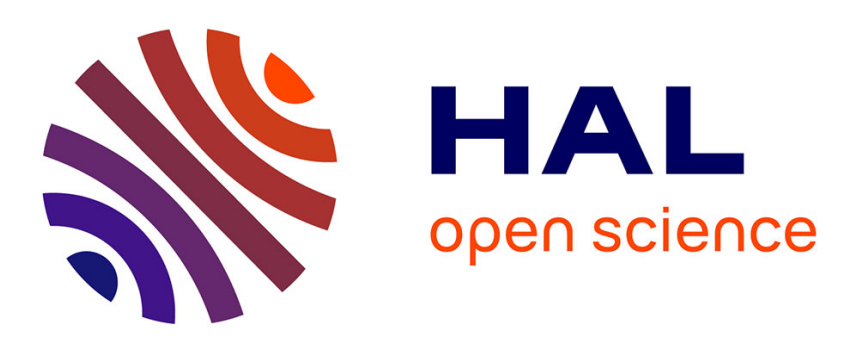

\title{
A local adjustment strategy for the initialization of dynamic causal modelling to infer effective connectivity in brain epileptic structures
}

Wentao Xiang, Ahmad Karfoul, Huazhong Shu, Régine Le Bouquin Jeannès

\section{- To cite this version:}

Wentao Xiang, Ahmad Karfoul, Huazhong Shu, Régine Le Bouquin Jeannès. A local adjustment strategy for the initialization of dynamic causal modelling to infer effective connectivity in brain epileptic structures. Computers in Biology and Medicine, 2017, 84, pp.30 - 44. 10.1016/j.compbiomed.2017.03.006 . inserm-01498128

\section{HAL Id: inserm-01498128 https://www.hal.inserm.fr/inserm-01498128}

Submitted on 29 Mar 2017

HAL is a multi-disciplinary open access archive for the deposit and dissemination of scientific research documents, whether they are published or not. The documents may come from teaching and research institutions in France or abroad, or from public or private research centers.
L'archive ouverte pluridisciplinaire HAL, est destinée au dépôt et à la diffusion de documents scientifiques de niveau recherche, publiés ou non, émanant des établissements d'enseignement et de recherche français ou étrangers, des laboratoires publics ou privés. 


\title{
A local adjustment strategy for the initialization of dynamic causal modelling to infer effective connectivity in brain epileptic structures
}

\author{
Wentao Xiang, ${ }^{\mathrm{a}, \mathrm{b}, \mathrm{d}}$, Ahmad Karfoul ${ }^{\mathrm{a}, \mathrm{b}}$, Huazhong Shu ${ }^{\mathrm{c}, \mathrm{d}}$, Régine Le Bouquin \\ Jeannès ${ }^{\mathrm{a}, \mathrm{b}, \mathrm{d}, *}$ \\ ${ }^{a}$ INSERM, U1099, Rennes, 35000, France \\ ${ }^{b}$ Université de Rennes 1, LTSI, Rennes, 35000, France \\ ${ }^{c}$ Laboratory of Image Science and Technology (LIST), School of Computer Science and \\ Engineering, Southeast University, Nanjing, 210096, China \\ ${ }^{d}$ Centre de Recherche en Information Biomédicale sino-français (CRIBs), 35000, France
}

\begin{abstract}
This paper addresses the question of effective connectivity in the human cerebral cortex in the context of epilepsy. Among model based approaches to infer brain connectivity, spectral Dynamic Causal Modelling is a conventional technique for which we propose an alternative to estimate cross spectral density. The proposed strategy we investigated tackles the sub-estimation of the free energy using the well-known variational Expectation-Maximization algorithm highly sensitive to the initialization of the parameters vector by a permanent local adjustment of the initialization process. The performance of the proposed strategy in terms of effective connectivity identification is assessed using simulated data generated by a neuronal mass model (simulating unidirectional and bidirectional flows) and real epileptic intracerebral Electroencephalographic signals. Results show the efficiency of proposed approach compared to the conventional Dynamic Causal Modelling and the one wherein a deterministic annealing scheme is employed.

Keywords: Effective connectivity; Dynamic causal modelling; Physiology based model; Epilepsy; Intracerebral EEG
\end{abstract}

\footnotetext{
*Corresponding author. Tel.: +33-2-23236919. Fax: +33-2-23236917.

Email address: regine.le-bouquin-jeannes@univ-rennes1.fr (Régine Le Bouquin Jeannès)
}

Preprint submitted to Computers in Biology and Medicine 


\section{Introduction}

Epilepsy is one of the most common neuronal diseases and concerns about $1 \%$ of the population. It results in a temporary dysfunction of the electrical brain activity, the epileptic seizure, arising from sudden abnormal electric discharges, known as paroxysmal discharges. These discharges occur repeatedly in one or several brain regions. Depending on the involved cortical areas, the clinical symptoms associated with the epileptic discharges vary from one patient to another and can lead to physical and mental impairments. The majority of epileptic patients can be successfully treated with drugs, which prevent the occurrence of epileptic seizures or attenuate their frequency. Now, in case of drugresistant epilepsies a surgical intervention is required to remove the epileptogenic zone (EZ) and consequently stop, or at least attenuate, the occurrence of seizures, under the constraint that post-surgical deficits are limited [1]. In practice, seizure activities are not systematically limited to the EZ and are distributed or modulated in distal and distinct brain structures. Hence, identifying the brain structure (network) responsible for the seizure onset is crucial in a pre-surgery phase even if it stands as a difficult task. The complexity of the problem relies on the fact that the cerebral network causing the epileptic seizure is patientdependent and the relationship between this network and the EZ is still not controlled. Compared to neuroimaging techniques (MRI, PET), intracerebral Electroencephalography (iEEG) is the most suited technique to characterize the rapid dynamics in a seizure activity thanks to its high temporal resolution, which permits to capture very fast neuronal dynamics related to the epileptic seizure. Therefore, this technique is considered as gold standard for EZ identification and allows a more precise brain exploration in spite of its invasiveness. Causal influences in-between network's nodes (cortical regions) are crucial in identifying the EZ. Characterizing the propagation information inside the brain refers to effective connectivity [2], i.e. to the causal effects exerted by one neural system over another one.

Quantifying effective connectivity goes back to the pioneer work of Wiener 
in 1956 who recognized the importance of temporal ordering in identifying the direction of the information flow [3]. Later, in 1969, Granger [4] gave the logarithmic implementation of Wiener's idea in the context of AutoRegressive (AR) models of stochastic processes with an application to econometric. Since then several causality measures have been developed such as directed coherence [5], frequency Geweke's Causality [6] and directed transfer function [7] to cite a few. Besides, other approaches in the scope of information theory have also been considered to infer effective connectivity such as transfer entropy [8] However, the aforementioned methods are known to be model-free techniques as no assumption on any specific underlying physiological model or prior knowledge concerning spatial or temporal underlying relationships is made. Therefore, considering such assumption would be relevant to understand how connected brain regions are coupled for a specific function generation such as epileptic seizure onset 9 .

Dynamic Causal Modelling (DCM) [10] has been proposed as a powerful tool to quantify effective connectivity. It is a model-based method, which consists in looking for the best model underlying the observed data. Inferring the best model is performed in a Bayesian model selection framework where each model structure corresponds to an alternative hypothesis about how the observed data are generated. The best model in a Bayesian framework is the one with the maximum model evidence (i.e. the log probability of the data given the model being tested) among all plausible models in the predefined model space. It is worth mentioning that the model space is defined according to the plausible generative models that could interpret the observed data. Generally, a state space representation consists of a state representation combined with a linear/nonlinear function that maps the state representation's inputs to the observation space (outputs). In the context of fMRI 11] this state representation is the well-known motion equation while it stands for a neural mass or field model when EEG or MEG data are observed [12. Furthermore, DCM is used to interpret both real and complex-valued data features. More particularly, Friston et al. 13. have proposed DCM for complex-valued data features based 
on Power Spectral Density (PSD) of local field potential data. This is performed by resorting to a linearization of the generative model around its steady state [14, 15. Recently, DCM gains more interest in quantifying effective connectivity in the context of epilepsy. Therefore, several models have been proposed as generative of the epileptic signals such as neural mass-like models [16] and cortical microcircuit ones [17, 18]. Typically, the Variational Bayes (VB) scheme [19] is used to estimate the evidence of each model defined in the model space. From a numerical point of view, the model evidence is expressed in terms of free energy and it is then computed by maximizing, under some assumptions, the latter [20. Generally, the well-known variational Expectation-Maximization (EM) algorithm is used to do such maximization. However, despite its efficiency, the EM algorithm and its variational version are highly sensitive to the initialization which results in potential sub-estimation of the model evidence. A Deterministic Annealing (DA) scheme [21, 22] and Markov chain Monte Carlo samplers [23] have been proposed to deal with such an issue. The key idea underlying this DA scheme is the use of a posterior parametrized by a "temperature" parameter to control the annealing process included in the Bayesian/variational EM method. More particularly, the maximization of the log-likelihood function is reformulated as minimizing the thermodynamic free energy based on statistical mechanics analogy to add temperature parameter [21, 22]. This DA scheme tracks the optimum of the considered objective function from high temperature wherein the objective function is smoothed (i.e. it has one global optimum) to low temperature wherein the shape of the objective function gradually approaches the one of the original objective function.

In this paper a local adjustment strategy tackling the initialization issue of the EM algorithm in the context of DCM and denoted by L-DCM is proposed. It is a generalization of our earlier work [16] towards a robust estimation of the model evidence. The proposed strategy is based on an adjustment of the initial parameters in the variational EM algorithm, locally, using the current estimation of the model parameters. The efficiency of the proposed L-DCM is evaluated in the context of simulated and real iEEG recordings in the context of drug-resistant 


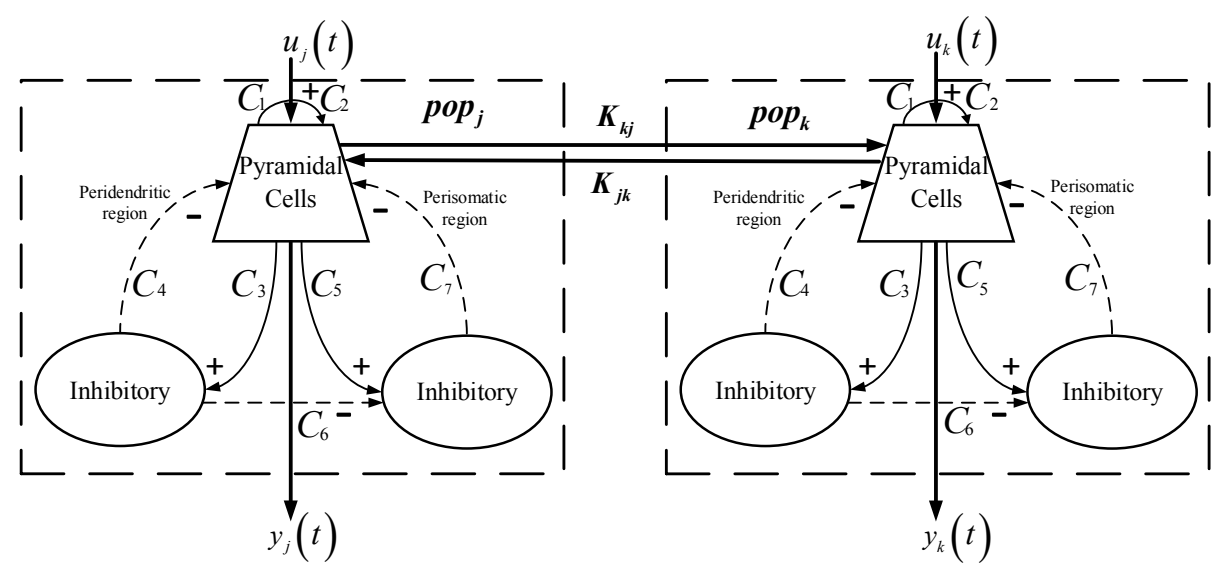

Fig. 1. Two potentially coupled populations $\operatorname{pop}_{j}$ and $p_{0} p_{k}[16]$.

epilepsy. Furthermore, the behaviour of the proposed strategy is compared to both the conventional DCM and the DCM when the DA scheme is employed [22, noted from now on as D-DCM. It is noteworthy that the simulated iEEG signals are generated using a physiology-based model of neural mass populations 24] developed in our team.

\section{Physiology-based model}

The electrical activities of potentially coupled brain regions are simulated using nonlinear time continuous Stochastic Differential Equations (SDE) according to 24]. The output of a neuronal population is seen as a nonlinear transformation of the local mean field activity by a quasi-static transfer function (see 24 for more details). Each neural population consists of three subpopulations, a major one standing for excitatory interneurons (main pyramidal cells) and two sub-populations corresponding to inhibitory interneurons, that mutually interact by positive or negative feedback via intrinsic coupling parameters $C_{i}, i=1, \ldots, 7$ as depicted in Fig. 1. Considering two populations pop $_{l}, l \in\{j, k\}$, each one is modeled using the above-mentioned neural-mass model and the dynamic of the hidden states of each population is mathematically described by a set of 16 one-order differential equations as follows: 


$$
\begin{aligned}
\dot{x}_{i, l}(t)= & x_{i+5, l}(t) \quad i=1,2, \ldots, 5 \\
\dot{x}_{6, l}(t)= & A_{l} a S\left(x_{13, l}(t)\right)-2 a x_{6, l}(t)-a^{2} x_{1, l}(t) \\
\dot{x}_{7, l}(t)= & A_{l} a C_{2} S\left(x_{14, l}(t)\right)-2 a x_{7, l}(t)-a^{2} x_{2, l}(t) \\
& +A_{l} a\left(u_{l}(t)+K_{l l^{\prime}} x_{11, l^{\prime}}(t)\right) \\
\dot{x}_{8, l}(t)= & B_{l} b C_{4} S\left(x_{15, l}(t)\right)-2 b x_{8, l}(t)-b^{2} x_{3, l}(t) \\
\dot{x}_{9, l}(t)= & G_{l} g C_{7} S\left(x_{16, l}(t)\right)-2 g x_{9, l}(t)-g^{2} x_{4, l}(t) \\
\dot{x}_{10, l}(t)= & B_{l} b S\left(x_{15, l}(t)\right)-2 b x_{10, l}(t)-b^{2} x_{5, l}(t) \\
\dot{x}_{11, l}(t)= & x_{12, l}(t) \\
\dot{x}_{12, l}(t)= & A_{l} a_{d} S\left(x_{13, l}(t)\right)-2 a_{d} x_{12, l}(t)-a_{d}^{2} x_{11, l}(t) \\
\dot{x}_{13, l}(t)= & x_{7, l}(t)-x_{8, l}(t)-x_{9, l}(t) \\
\dot{x}_{14, l}(t)= & C_{1} x_{6, l}(t) \\
\dot{x}_{15, l}(t)= & C_{3} x_{6, l}(t) \\
\dot{x}_{16, l}(t)= & C_{5} x_{6, l}(t)-C_{6} x_{10, l}(t)
\end{aligned}
$$

where $S(x(t))=\frac{2 e_{0}}{1+\exp \left(r_{0}\left(V_{0}-x(t)\right)\right)}$ is the sigmoid function. The noise input of each population, denoted by $u_{l}(t), l \in\{j, k\}$, summarizes all exogenous contributions and is given by: $u_{l}(t)=90+30 w_{l}(t)$, where $w_{l}(t) \sim \mathcal{N}\left(0, \alpha_{l}\right)$, such that $w_{j}(t)$ and $w_{k}(t)$ are independent [24]. The directional connection from $p^{\prime} p_{l}$ to pop $_{l^{\prime}}$, which reflects the number of axonal links, is characterized by the coupling parameter $K_{l^{\prime} l},\left\{l, l^{\prime}\right\} \in\{j, k\}, l^{\prime} \neq l$. According to the coupling parameters $\left\{K_{k j}, K_{j k}\right\}$ (see Fig. 1), a model space of four possible model structures describing the relation between the two populations under test can be figured out:

M1: No connection $\left(K_{k j}=K_{j k}=0\right)$;

M2: Unidirectional relation from $\operatorname{pop}_{j}$ to $\operatorname{pop}_{k}\left(K_{k j}>0, K_{j k}=0\right)$;

M3: Unidirectional relation from рор $_{k}$ to рор $_{j}\left(K_{j k}>0, K_{k j}=0\right)$;

M4: Bidirectional connection $\left(K_{j k}>0, K_{k j}>0\right)$.

The parameters controlling the intrinsic activity of a given population ${ } o_{l}$ (normal background versus epileptic state) are given by the parameters vector 
$\boldsymbol{\theta}_{l}=\left[\theta_{A_{l}}, \theta_{B_{l}}, \theta_{G_{l}}, \theta_{K_{l l^{\prime}}}, \theta_{\alpha_{l}}\right]^{\top}$, where the superscript ${ }^{\top}$ stands for the transpose operator and the parameter $\theta_{K_{l l^{\prime}}}$ reflects a possible causal effect from pop $_{l^{\prime}}$ to pop $_{l}, l^{\prime} \neq l$. In case of two independent populations (i.e. the case of model M1), $\theta_{K_{l l^{\prime}}}$ is dropped and we write $\boldsymbol{\theta}_{l}=\left[\theta_{A_{l}}, \theta_{B_{l}}, \theta_{G_{l}}, \theta_{\alpha_{l}}\right]^{\top}$ instead. Consequently, $\boldsymbol{\theta}=\left[\boldsymbol{\theta}_{j}^{\top} \boldsymbol{\theta}_{k}^{\top}\right]^{\top}$ denotes the vector of all model parameters to be estimated. Prior values of the components of the vector $\boldsymbol{\theta}_{l}$ are given in Table 1 . The other set of parameters figured in (1), i.e. $\left\{a, a_{d}, b, g, r_{0}, V_{0}, e_{0}\right\}$, is assumed to be constant as in [24]. Regarding the population output, $y_{l}(t), l \in\{j, k\}$, it reflects the membrane potential of pyramidal cells, which can be considered as a zero-mean iEEG signal, and is computed as the difference between $x_{13, l}(t)$ and its time average $\overline{x_{13, l}}, l \in\{j, k\}$ :

$$
y_{l}(t)=x_{13, l}(t)-\overline{x_{13, l}}
$$

\section{Methodology}

This section is devoted to describe the proposed L-DCM algorithm with the aim of getting the best model evidence by maximizing the PSD of the observed data. Both techniques, the proposed L-DCM and the conventional DCM, employ the VB scheme for such maximization. However, the proposed L-DCM method further includes a local adjustment strategy to well-condition the starting point of the variational EM algorithm in the maximization step.

Table 1. Prior on model parameters for $\operatorname{pop}_{l}, \ell \in\{i, j\}[16]$

\begin{tabular}{ll}
\hline Parameters & Prior Distribution \\
\hline Synaptic gain excitation $A_{l}$ & $A_{l}=5 \exp \left(\theta_{A_{l}}\right), \theta_{A_{l}} \sim \mathcal{N}(0,1 / 16)$ \\
Dendritic slow inhibition $B_{l}$ & $B_{l}=3 \exp \left(\theta_{B_{l}}\right), \theta_{B_{l}} \sim \mathcal{N}(0,1 / 16)$ \\
Somatic fast inhibition $G_{l}$ & $G_{l}=20 \exp \left(\theta_{G_{l}}\right), \theta_{G_{l}} \sim \mathcal{N}(0,1 / 16)$ \\
Extrinsic connections $K_{l l^{\prime}}$ & $K_{l l^{\prime}}=500 \exp \left(\theta_{K_{l l^{\prime}}}\right), \theta_{K_{l l^{\prime}}} \sim \mathcal{N}(0,1)$ \\
Input white noise variance $\alpha_{l}$ & $\alpha_{l}=\exp \left(\theta_{\alpha_{l}}\right), \theta_{\alpha_{l}} \sim \mathcal{N}(0,1 / 64)$ \\
\hline
\end{tabular}




\subsection{Towards a PSD maximization}

Initially, DCM and L-DCM optimize the model structure by estimating its parameters based on the computation of the data's PSD. For two potentially coupled populations, the physiology-based model described by (1) and (2) can be represented in a set of $2 \times 16$ first-order differential equations and a two-component output vector summarized in a state space representation as:

$$
\left\{\begin{array}{l}
\dot{\boldsymbol{x}}(t)=\boldsymbol{f}(\boldsymbol{x}(t), \boldsymbol{\theta})+\boldsymbol{D u}(t) \\
\boldsymbol{y}(t)=\boldsymbol{L}(\boldsymbol{x}(t)-\overline{\boldsymbol{x}})
\end{array}\right.
$$

where $\overline{\boldsymbol{x}}$ is the time average of $\boldsymbol{x}(t)$ and

$$
\begin{aligned}
& \boldsymbol{x}_{l}(t)=\left[x_{1, l}(t), \ldots, x_{16, l}(t)\right]^{\top} \in \Re^{16 \times 1} \\
& \boldsymbol{x}(t)=\left[\boldsymbol{x}_{j}^{\top}(t) \boldsymbol{x}_{k}^{\top}(t)\right]^{\top} \in \Re^{32 \times 1}, \boldsymbol{f}_{l}\left(\boldsymbol{x}(t), \boldsymbol{\theta}_{l}\right) \in \Re^{16 \times 1} \\
& \boldsymbol{f}(\boldsymbol{x}(t), \boldsymbol{\theta})=\left[\boldsymbol{f}_{j}^{\top}\left(\boldsymbol{x}(t), \boldsymbol{\theta}_{j}\right) \boldsymbol{f}_{k}^{\top}\left(\boldsymbol{x}(t), \boldsymbol{\theta}_{k}\right)\right]^{\top} \in \Re^{32 \times 1} \\
& \boldsymbol{D} \in \Re^{32 \times 2},(\boldsymbol{D})_{7,1}=A_{j} a,(\boldsymbol{D})_{23,2}=A_{k} a \\
& \boldsymbol{L} \in \Re^{2 \times 32},(\boldsymbol{L})_{1,13}=1,(\boldsymbol{L})_{2,29}=1 \\
& \boldsymbol{u}(t)=\left[u_{j}(t), u_{k}(t)\right]^{\top} \in \Re^{2 \times 1}, \boldsymbol{y}(t)=\left[y_{j}(t), y_{k}(t)\right]^{\top} \in \Re^{2 \times 1}
\end{aligned}
$$

where $(\boldsymbol{Z})_{k_{1}, k_{2}}$ denotes the $\left(k_{1}, k_{2}\right)$-th entry of the matrix $\boldsymbol{Z} \in\{\boldsymbol{D}, \boldsymbol{L}\}$. Both the input and the output matrices, namely $\boldsymbol{D}$ and $\boldsymbol{L}$, respectively contain only two non-zero entries. Due to the sigmoid function defined previously, the function $\boldsymbol{f}(\boldsymbol{x}(t), \boldsymbol{\theta})$ is nonlinear and, consequently, the system output $\boldsymbol{y}(t)$ results from a nonlinear transformation of the input $\boldsymbol{u}(t)$. Therefore a linearization of (3) around the equilibrium state $\boldsymbol{x}_{0}(\boldsymbol{\theta}) \in \Re^{32 \times 1}$ is performed. Note that the equilibrium point, if it exists, is defined as the solution of the equation $\mathbf{0}=\boldsymbol{f}\left(\boldsymbol{x}_{0}, \boldsymbol{\theta}\right)+\boldsymbol{D} \boldsymbol{u}_{0}(t)$ with $\boldsymbol{u}_{0}(t)=[90,90]^{\top}[24$, otherwise the steady state trajectory generally stays on a limit cycle, and we set $\boldsymbol{x}_{0} \simeq \overline{\boldsymbol{x}}[16$. Following [14, the system in (3) is linearized using first-order Taylor expansion as follows:

$$
\left\{\begin{aligned}
\delta \dot{\boldsymbol{x}}(t) & =\Im\left(\boldsymbol{x}_{0}\right) \delta \boldsymbol{x}(t)+\boldsymbol{D}_{1} \boldsymbol{w}(t) \\
\boldsymbol{y}(t) & =\boldsymbol{L} \delta \boldsymbol{x}(t)
\end{aligned}\right.
$$


where $\delta \boldsymbol{x}(t)=\boldsymbol{x}(t)-\boldsymbol{x}_{0}, \Im\left(\boldsymbol{x}_{0}\right)=\left.\frac{\partial \boldsymbol{f}(\boldsymbol{x}(t), \boldsymbol{\theta})}{\partial \boldsymbol{x}(t)}\right|_{\boldsymbol{x}(t)=\boldsymbol{x}_{0}} \in \Re^{32 \times 32}$ is the Jacobian matrix of $\boldsymbol{f}(\cdot, \boldsymbol{\theta})$ computed at $\boldsymbol{x}=\boldsymbol{x}_{0}, \boldsymbol{D}_{1}=30 \times \boldsymbol{D}$ and $\boldsymbol{w}(t)=\left[w_{j}(t), w_{k}(t)\right]^{\top}$. Considering the Laplace transforms in (4), we get:

$$
\left\{\begin{array}{c}
s \delta \boldsymbol{X}(s)=\Im\left(\boldsymbol{x}_{0}\right) \delta \boldsymbol{X}(s)+\boldsymbol{D}_{1} \boldsymbol{W}(s) \\
\boldsymbol{Y}(s)=\boldsymbol{L} \delta \boldsymbol{X}(s)=\boldsymbol{H}(s, \boldsymbol{\theta}) \boldsymbol{W}(s)
\end{array}\right.
$$

where $\boldsymbol{H}(s, \boldsymbol{\theta})=\boldsymbol{L}\left(s \boldsymbol{I}-\Im\left(\boldsymbol{x}_{0}\right)\right)^{-1} \boldsymbol{D}_{1} \in \Re^{2 \times 2}$ is the frequency transfer function, $s=\sqrt{-1} \omega, \omega=2 \pi \nu$ ( $\nu$ is the frequency bin) and $\boldsymbol{I}$ is the identity matrix. The analytical expression of the PSD of the signal vector $\boldsymbol{y}(t)$ denoted by $\boldsymbol{g}(\nu, \boldsymbol{\theta}) \in \Re^{2 \times 2}$ can be quantified by the transform function $\boldsymbol{H}(s, \boldsymbol{\theta})$ and the input PSD denoted by $\alpha_{l}, l \in\{j, k\}$, such that:

$$
\boldsymbol{g}(\nu, \boldsymbol{\theta})=\boldsymbol{Y}(s) \times \boldsymbol{Y}^{\mathrm{H}}(s)=\boldsymbol{H}(s, \boldsymbol{\theta})\left[\begin{array}{cc}
\alpha_{j} & 0 \\
0 & \alpha_{k}
\end{array}\right] \boldsymbol{H}^{\mathrm{H}}(s, \boldsymbol{\theta})
$$

where the superscript ${ }^{\mathrm{H}}$ is the conjugate transpose operator. Therefore, given the parameters $\boldsymbol{\theta}$ of the generative model, it is easy to compute the analytical expression of the PSD [15]. In practice, the PSD is computed using the SPM spectral toolbox with a 12-pole AR process [15]. In this case, this PSD, called measured PSD, is denoted by $\tilde{\boldsymbol{g}}(\nu) \in \Re^{2 \times 2}$. Thus, the following equation holds:

$$
\tilde{\boldsymbol{g}}(\nu)=\boldsymbol{g}(\nu, \boldsymbol{\theta})+\boldsymbol{\varepsilon}(\nu)
$$

where $\varepsilon(\nu) \in \Re^{2 \times 2}$ is the sampling error matrix such that $\varepsilon(\nu) \sim \mathcal{N}\left(\mathbf{0}, \boldsymbol{\Sigma}_{\nu}(\lambda)\right)$, $\boldsymbol{\Sigma}_{\boldsymbol{\nu}}$ stands for the covariance matrix and $\lambda$ denotes the vector of channels variances. According to (7), it is obvious that estimating the PSD is, up to some sampling error, directly linked to the estimation of the optimal model parameters. An optimal model, among all plausible models in the predefined model space, is designed as the one with the maximum PSD, (i.e. maximal $\tilde{\boldsymbol{g}}(\nu)$ ). Maximizing the measured PSD is typically performed with a VB scheme using the variational EM algorithm as extensively detailed in the next subsection. Defining an optimal model structure underlying the observed signals is performed by maximizing the 
likelihood of $\tilde{\boldsymbol{g}}(\nu)$ given a model structure, noted by $p(\tilde{\boldsymbol{g}}(\nu) \mid \mathrm{M} m)$. However, since $\boldsymbol{g}(\nu, \boldsymbol{\theta})$ and consequently $\tilde{\boldsymbol{g}}(\nu)$ are nonlinear in the parameter vector $\boldsymbol{\theta}$ due to the sigmoid function, the likelihood $p(\tilde{\boldsymbol{g}}(\nu) \mid \mathrm{M} m)$ is not conjugate to the Gaussian priors on the parameters [19]. Hence, a classical Bayesian estimation of the parameters requires computing an integral of the probability density which is, in turn, a hard task especially for high dimensional case. To cope with this issue, the VB scheme has been proposed [16] wherein the log-likelihood, $\ln p(\tilde{\boldsymbol{g}}(\nu) \mid \mathrm{M} m)$, is indirectly approximated by the so-called free energy [20]:

$$
\ln p(\tilde{\boldsymbol{g}}(\nu) \mid \mathrm{M} m)=F_{m}+K L(q(\boldsymbol{\theta}), p(\boldsymbol{\theta} \mid \tilde{\boldsymbol{g}}(\nu), \mathrm{M} m))
$$

In the above equation, $K L$ is the Kullback-Leibler divergence, $p(\boldsymbol{\theta} \mid \tilde{\boldsymbol{g}}(\nu), \mathrm{M} m)$ is the posterior parameter distribution of the parameters vector $\boldsymbol{\theta}, q(\boldsymbol{\theta}) \sim$ $\mathcal{N}\left(\hat{\boldsymbol{\theta}}, \boldsymbol{\Sigma}_{\hat{\boldsymbol{\theta}}}\right)$ is the approximate posterior distribution of $\boldsymbol{\theta}$ which follows a normal distribution with mean vector $\hat{\boldsymbol{\theta}}$ and covariance matrix $\boldsymbol{\Sigma}_{\hat{\boldsymbol{\theta}}}$. According to (8), it is obvious that maximizing the $\log$-likelihood, $\ln p(\tilde{\boldsymbol{g}}(\nu) \mid \mathrm{M} m)$, is equivalent to maximizing the free energy given a model structure, $\mathrm{M} m$. Note that maximizing the free energy implies a minimization of the Kullback-Leibler divergence term. Maximizing the free energy, $F_{m}$, is usually done in an iterative way using the variational $\mathrm{EM}$ algorithm whose main steps are summarized below [20]:

Initialization $\boldsymbol{\theta}_{0}=\left[\boldsymbol{\theta}_{0, j}^{\top} \boldsymbol{\theta}_{0, k}^{\top}\right]^{\top}, \hat{\boldsymbol{\theta}}=\boldsymbol{\theta}_{0}, \boldsymbol{\lambda}, \boldsymbol{\Sigma}_{\hat{\boldsymbol{\theta}}}$

Repeat until convergence or a maximum no. of iterations is reached

$$
\begin{aligned}
& \text { E-Step : }\left(\hat{\boldsymbol{\theta}}, \boldsymbol{\Sigma}_{\hat{\boldsymbol{\theta}}}\right)=\max _{(\text {fix } \boldsymbol{\lambda})} F_{m}\left(\hat{\boldsymbol{\theta}}, \boldsymbol{\lambda}, \boldsymbol{\Sigma}_{\hat{\boldsymbol{\theta}}}\right) \\
& \mathrm{M}-\text { Step }: \quad \boldsymbol{\lambda}=\max _{\left(\text {fix } \hat{\boldsymbol{\theta}}, \boldsymbol{\Sigma}_{\hat{\boldsymbol{\theta}}}\right)} F_{m}\left(\hat{\boldsymbol{\theta}}, \boldsymbol{\lambda}, \boldsymbol{\Sigma}_{\hat{\boldsymbol{\theta}}}\right)
\end{aligned}
$$

end

$$
F_{m}=F_{m}\left(\hat{\boldsymbol{\theta}}, \boldsymbol{\lambda}, \boldsymbol{\Sigma}_{\hat{\boldsymbol{\theta}}}\right)
$$

In the above pseudo-code, recall that $\boldsymbol{\lambda}$ is the vector of channels variances, $\boldsymbol{\theta}_{0}$ is the initial vector for variational EM algorithm. 


\subsection{The proposed strategy}

The variational EM algorithm is known to be highly sensitive to the initialization and so it may lead to sub-estimation of the free energy and consequently to a misestimation of the model parameters. To circumvent this problem, a local adjustment initialization strategy is adopted in this paper. When applied this local adjustment strategy for the DCM technique, it gives rise to the L-DCM method. The latter mainly consists in refining the variational EM estimation by employing several sweeps of the variational EM algorithm with initial guess denoted by $\boldsymbol{\theta}_{0}^{(r)}$ adjusted for each $r$-th sweep. To improve the estimation quality of the PSD for each population involved in the model structure being tested, an adequate criterion must be introduced. To this end, we first define, for each population, a threshold, $T h_{l l}$, and a prediction error, $E r_{l l}, l \in\{j, k\}:$

$$
\begin{aligned}
& T h_{l l}=\frac{1}{N_{\nu}} \sum_{\nu=\nu_{a}^{(l)}}^{\nu_{b}^{(l)}} \gamma^{2} \tilde{g}_{l l}(\nu)^{2} \\
& E r_{l l}=\frac{1}{N_{\nu}} \sum_{\nu=\nu_{a}^{(l)}}^{\nu_{b}^{(l)}}\left(g_{l l}(\nu, \hat{\theta})-\tilde{g}_{l l}(\nu)\right)^{2}
\end{aligned}
$$

$g_{l l}(\nu, \hat{\boldsymbol{\theta}})$ stands for the estimation of the analytical PSD obtained by substituting the model parameter estimate $\hat{\boldsymbol{\theta}}$ using the variational EM algorithm in $(9)$ and $N_{\nu}$ is the sum of frequency bins in the frequency interval $\left[\nu_{a}^{(l)}, \nu_{b}^{(l)}\right]$ associated with the $l$-th population and comprising the spectral power features of interest, where $\nu_{a}^{(l)}=\nu_{m}^{(l)}-10, \nu_{b}^{(l)}=\nu_{m}^{(l)}+10$ with $\nu_{m}^{(l)}$ standing for the frequency bin corresponding to the maximum measured PSD. Also, $\gamma$ stands for a weighting coefficient (confidence parameter) fixed empirically to $10 \%$ to guarantee an acceptable variation in a limited frequency band interval (including, according to (10), the maximum measured PSD), between the measured PSD $\tilde{g}_{l l}(\nu)$ and the estimated one $g_{l l}(\nu, \hat{\boldsymbol{\theta}})$ computed according to (6) using the estimate $\hat{\boldsymbol{\theta}}$. According to (10), a good estimation of the PSD $g_{l l}(\nu, \hat{\boldsymbol{\theta}})$ is obtained when $E r_{l l} \leq T h_{l l}, l \in\{j, k\}$. However, in case this inequality is not fulfilled, three situations can be figured out: situation (1): $E r_{j j} \leq T h_{j j}, E r_{k k}>T h_{k k}$; situation (2): $E r_{j j}>T h_{j j}, E r_{k k} \leq T h_{k k}$; situation 
(3): $E r_{j j}>T h_{j j}, E r_{k k}>T h_{k k}$. To cope with those situations, the proposed L-DCM algorithm refines the variational EM estimation results by performing few supplementary well-conditioned variational EM sweeps. More precisely, the initial guess of each sweep is adjusted by taking into account i) the estimation results of the previous variational EM sweep, ii) the encountered situation (i.e. (1) or (2) or (3)) and iii) the model structure, $\mathrm{M} m, m \in\{1,2,3,4\}$ under consideration. The key idea of the adjustment strategy consists in identifying, for the $(r+1)$-th variational EM sweep, $r \in\{1,2, \ldots, R\}$ where $R$ is the maximum number of sweeps, the vector component(s) $\hat{\boldsymbol{\theta}}_{l}^{(r)}, l \in\{j, k\}$, of $\hat{\boldsymbol{\theta}}^{(r)}$ responsible for the given situation (i.e. (1) or (2) or (3)). The identified component(s) is(are) then adjusted by adding a random vector dropping from zero-mean normal distribution with predefined variance. The $(r+1)$-th variational EM sweep is run with the initial point $\boldsymbol{\theta}_{0}^{(r+1)}$ equal to the adjusted model parameter(s) vector. For the sake of clarity, let us consider the two following illustrative examples.

Example 1: Consider the case of maximizing the free energy given the model structure M1. Assume that the situation (1) ( i.e. $E r_{j j} \leq T h_{j j}, E r_{k k}>T h_{k k}$ ) occurs for the $r$-th variational EM sweep. In this case, the initial parameter concerning the vector component $\hat{\boldsymbol{\theta}}_{j}^{(r)}$ for the first population $\operatorname{pop}_{j}$ is considered as a suitable starting point, whereas the one related to the second population (i.e. $\operatorname{pop}_{k}$ ) is not so and therefore should be adjusted. Consequently, the initial guess to estimate the model parameter vector for the $(r+1)$-th variational EM sweep is defined as follows:

$$
\boldsymbol{\theta}_{0}^{(r+1)}=\left[\begin{array}{c}
\boldsymbol{\theta}_{0, j}^{(r+1)} \\
\boldsymbol{\theta}_{0, k}^{(r+1)}
\end{array}\right]=\left[\begin{array}{c}
\hat{\boldsymbol{\theta}}_{j}^{(r)} \\
\hat{\boldsymbol{\theta}}_{k}^{(r)}+\Delta \hat{\boldsymbol{\theta}}_{k}
\end{array}\right]
$$

where $\Delta \hat{\boldsymbol{\theta}}_{k} \sim \mathcal{N}\left(\mathbf{0}, \sigma^{2} \mathbf{I}\right), \boldsymbol{I}$ is the identity matrix and $\sigma^{2}$ is a data-dependent scalar to be determined. A detailed discussion regarding the choice of $\sigma^{2}$ is given in section 4 ,

Example 2: Consider now the case of maximizing the free energy given the model M2. Assume that situation (2) (i.e. $E r_{j j}>T h_{j j}, E r_{k k} \leq T h_{k k}$ ) 
Table 2. L-DCM adjustment strategy for different situations and different plausible models

\begin{tabular}{|c|c|c|c|}
\hline \multirow{2}{*}{ Model } & \multicolumn{3}{|c|}{ Strategies } \\
\hline & (1) & (2) & (3) \\
\hline M1 & $\begin{array}{l}\boldsymbol{\theta}_{0, j}^{(r+1)}=\hat{\boldsymbol{\theta}}_{j}^{(r)} \\
\boldsymbol{\theta}_{0, k}^{(r+1)}=\hat{\boldsymbol{\theta}}_{k}^{(r)}+\Delta \hat{\boldsymbol{\theta}}_{k}\end{array}$ & $\begin{array}{l}\boldsymbol{\theta}_{0, j}^{(r+1)}=\hat{\boldsymbol{\theta}}_{j}^{(r)}+\Delta \hat{\boldsymbol{\theta}}_{j} \\
\boldsymbol{\theta}_{0, k}^{(r+1)}=\hat{\boldsymbol{\theta}}_{k}^{(r)}\end{array}$ & $\begin{array}{l}\boldsymbol{\theta}_{0, j}^{(r+1)}=\hat{\boldsymbol{\theta}}_{j}^{(r)}+\Delta \hat{\boldsymbol{\theta}}_{j} \\
\boldsymbol{\theta}_{0, k}^{(r+1)}=\hat{\boldsymbol{\theta}}_{k}^{(r)}+\Delta \hat{\boldsymbol{\theta}}_{k}\end{array}$ \\
\hline M2 & $\begin{array}{l}\boldsymbol{\theta}_{0, j}^{(r+1)}=\hat{\boldsymbol{\theta}}_{j}^{(r)} \\
\boldsymbol{\theta}_{0, k}^{(r+1)}=\hat{\boldsymbol{\theta}}_{k}^{(r)}+\Delta \hat{\boldsymbol{\theta}}_{k}\end{array}$ & $\begin{array}{l}\boldsymbol{\theta}_{0, j}^{(r+1)}=\hat{\boldsymbol{\theta}}_{j}^{(r)}+\Delta \hat{\boldsymbol{\theta}}_{j} \\
\boldsymbol{\theta}_{0, k}^{(r+1)}=\hat{\boldsymbol{\theta}}_{k}^{(r)}+\Delta \hat{\boldsymbol{\theta}}_{k}\end{array}$ & $\begin{array}{l}\boldsymbol{\theta}_{0, j}^{(r+1)}=\hat{\boldsymbol{\theta}}_{j}^{(r)}+\Delta \hat{\boldsymbol{\theta}}_{j} \\
\boldsymbol{\theta}_{0, k}^{(r+1)}=\hat{\boldsymbol{\theta}}_{k}^{(r)}+\Delta \hat{\boldsymbol{\theta}}_{k}\end{array}$ \\
\hline M3 & $\begin{array}{l}\boldsymbol{\theta}_{0, j}^{(r+1)}=\hat{\boldsymbol{\theta}}_{j}^{(r)}+\Delta \hat{\boldsymbol{\theta}}_{j} \\
\boldsymbol{\theta}_{0, k}^{(r+1)}=\hat{\boldsymbol{\theta}}_{k}^{(r)}+\Delta \hat{\boldsymbol{\theta}}_{k}\end{array}$ & $\begin{array}{l}\boldsymbol{\theta}_{0, j}^{(r+1)}=\hat{\boldsymbol{\theta}}_{j}^{(r)}+\Delta \hat{\boldsymbol{\theta}}_{j} \\
\boldsymbol{\theta}_{0, k}^{(r+1)}=\hat{\boldsymbol{\theta}}_{k}^{(r)}\end{array}$ & $\begin{array}{l}\boldsymbol{\theta}_{0, j}^{(r+1)}=\hat{\boldsymbol{\theta}}_{j}^{(r)}+\Delta \hat{\boldsymbol{\theta}}_{j} \\
\boldsymbol{\theta}_{0, k}^{(r+1)}=\hat{\boldsymbol{\theta}}_{k}^{(r)}+\Delta \hat{\boldsymbol{\theta}}_{k}\end{array}$ \\
\hline M4 & $\begin{array}{l}\boldsymbol{\theta}_{0, j}^{(r+1)}=\hat{\boldsymbol{\theta}}_{j}^{(r)}+\Delta \hat{\boldsymbol{\theta}}_{j} \\
\boldsymbol{\theta}_{0, k}^{(r+1)}=\hat{\boldsymbol{\theta}}_{k}^{(r)}+\Delta \hat{\boldsymbol{\theta}}_{k}\end{array}$ & $\begin{array}{l}\boldsymbol{\theta}_{0, j}^{(r+1)}=\hat{\boldsymbol{\theta}}_{j}^{(r)}+\Delta \hat{\boldsymbol{\theta}}_{j} \\
\boldsymbol{\theta}_{0, k}^{(r+1)}=\hat{\boldsymbol{\theta}}_{k}^{(r)}+\Delta \hat{\boldsymbol{\theta}}_{k}\end{array}$ & $\begin{array}{l}\boldsymbol{\theta}_{0, j}^{(r+1)}=\hat{\boldsymbol{\theta}}_{j}^{(r)}+\Delta \hat{\boldsymbol{\theta}}_{j} \\
\boldsymbol{\theta}_{0, k}^{(r+1)}=\hat{\boldsymbol{\theta}}_{k}^{(r)}+\Delta \hat{\boldsymbol{\theta}}_{k}\end{array}$ \\
\hline
\end{tabular}

occurs for the $r$-th variational EM sweep. In this case, the initial parameter concerning the vector component $\hat{\boldsymbol{\theta}}_{j}^{(r)}$ for the population pop $_{j}$ is not suitable and therefore should be adjusted. Regarding the vector component $\hat{\boldsymbol{\theta}}_{k}^{(r)}$ for the second population, pop $_{k}$, it should be also adjusted since according to the model M2 assumption, $\operatorname{pop}_{j}$ carries out a causal effect on $\operatorname{pop}_{k}$. Consequently, the initial guess to estimate the model parameter vector for the $(r+1)$-th variational EM sweep is, in this case, defined as follows:

$$
\boldsymbol{\theta}_{0}^{(r+1)}=\left[\begin{array}{c}
\boldsymbol{\theta}_{0, j}^{(r+1)} \\
\boldsymbol{\theta}_{0, k}^{(r+1)}
\end{array}\right]=\left[\begin{array}{c}
\hat{\boldsymbol{\theta}}_{j}^{(r)}+\Delta \hat{\boldsymbol{\theta}}_{j} \\
\hat{\boldsymbol{\theta}}_{k}^{(r)}+\Delta \hat{\boldsymbol{\theta}}_{k}
\end{array}\right]
$$

Table 2 summarizes the proposed adjustment strategy of the proposed L-DCM approach for all critical situations and all plausible models in the defined model space. The flow chart of the L-DCM algorithm is displayed in Fig. 2

According to the proposed L-DCM approach, the best model Mm, $m \in$ $\{1,2,3,4\}$, underlying the observed data, is the one leading to the maximum free energy, $F_{m}, m \in\{1,2,3,4\}$, after well-conditioned variational EM sweeps. The maximum number $R$ of sweeps should be experimentally chosen such that we get an acceptable tradeoff between a moderate computational complexity and sufficient estimation accuracy. According to our studies, the choice $R=6$ fulfills the aforementioned tradeoff. Note that L-DCM can be switched immediately to DCM at $R=1$. Hence, DCM can be seen as a particular case of L-DCM. 


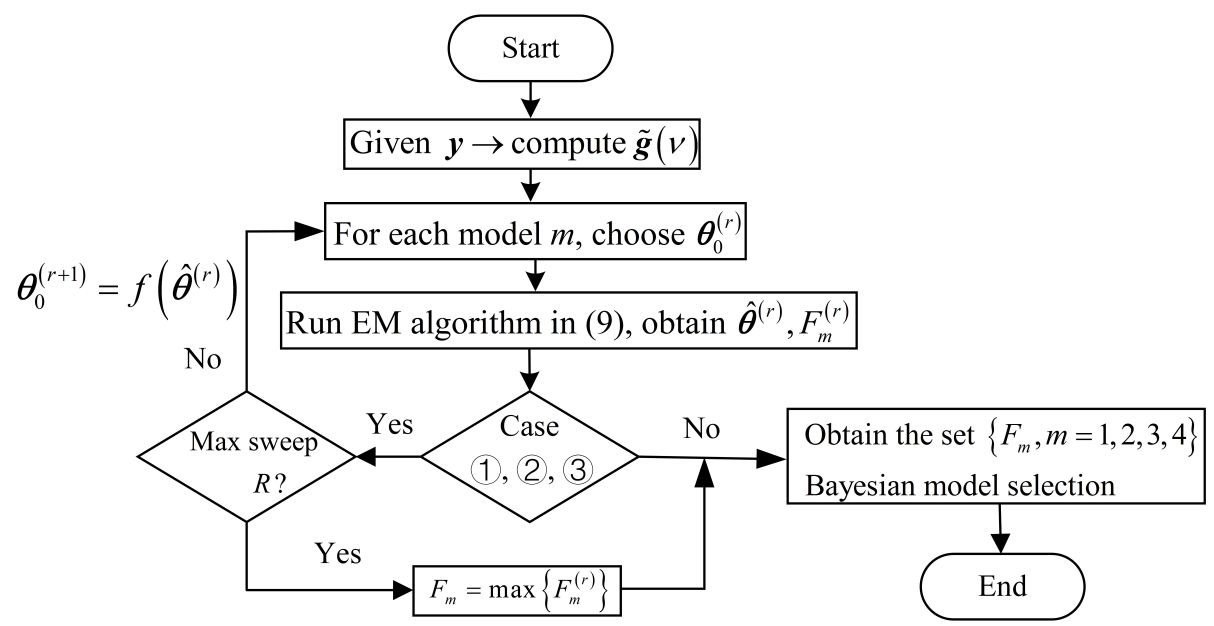

Fig. 2. Algorithm flow chart of L-DCM

\section{Results}

This section is devoted to provide comprehensive insight about the L-DCM performance in quantifying effective connectivity in epileptic neural populations. The behaviour of the L-DCM method is compared to the conventional DCM one [16] and its variant the D-DCM wherein the DA scheme [22] is employed. This comparative study is conducted firstly using realistic iEEG recordings generated according to the physiology-based model developed in our team [24]. Secondly, human epileptic iEEG signals are considered. It is worth mentioning that the use of simulated signals is two-fold. On the one hand, their characteristics are similar to those of real signals observed at the onset of epileptic seizures. On the other hand, they provide a ground truth regarding the model structure underlying the observed data, which is hardly accessible in real conditions.

\subsection{Simulated iEEG signals}

\subsubsection{Unidirectional flow}

Let us consider the model structure M2 reflecting the unidirectional flow from pop $_{j}$ to pop $_{k}$ which are assumed to be epileptic. This model is assumed to generate the observed iEEG signals (ground truth). According to (11), the 
proposed updating strategy of the model parameter at the $(r+1)$-th sweep, namely $\boldsymbol{\theta}_{0}^{r+1}$, depends on the variance $\sigma^{2}$, of the adjustment parameter $\Delta \hat{\boldsymbol{\theta}}_{k}$. Therefore a best choice of $\sigma^{2}$ regarding our considered application is discussed at first in this section. Then, the comparative study between the L-DCM, the DCM and the D-DCM methods is considered. Note that all aforementioned studies are conducted as a function of the coupling strength, $K_{k j}$, between the two populations. Therefore, three scenarios are considered throughout this section:

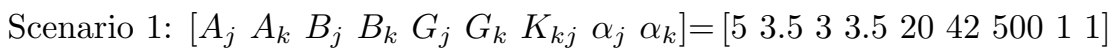

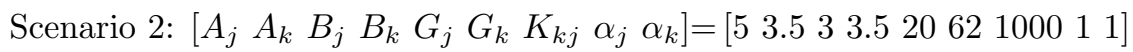

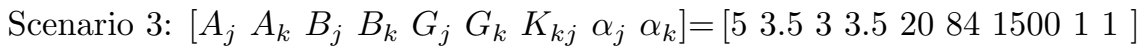
where the coupling strength (i.e. $K_{k j}$ ) is successively equal to 500, 1000 and 1500 from scenario 1 to scenario 3 . For each scenario, the set of parameters is chosen in such a way that the output $\boldsymbol{y}(t)$ reveals a $25 \mathrm{~Hz}$ sinusoidal activity comparable to the one of a real epileptic activity observed at the seizure onset (see Fig. 3 (a)).

\subsubsection{Choice of $\sigma^{2}$}

To investigate the influence of the parameter $\sigma^{2}$ on the free energy maximization in the L-DCM method, six different values of $\sigma^{2}$ are considered, $\sigma^{2} \in$ $\{0.05,0.1,0.15,0.2,0.8,1\}$. According to (11), a relatively low value of $\sigma^{2}$ is expected to provide well-conditioned initialization for the $(r+1)$-th sweep that is consistent with the results of the $(r)$-th one. However, a relatively high value of $\sigma^{2}$ (i.e. $\sigma^{2} \approx 1$ ) would result in inconsistent initialization between two successive sweeps. Fig. 4 shows the maximized free energy as a function of $\sigma^{2}$ for all defined scenarios (i.e. scenarios 1, 2 and 3). For reason of space, results regarding the values $\sigma^{2}=0.2$ and $\sigma^{2}=0.8$ were omitted since the results of the former were similar to the ones of $\sigma^{2}=0.15$ and the results of the latter were similar to that of $\sigma^{2}=1$. According to Fig. 4 the median values of the maximized free energy for all models tested in all scenarios show a higher free energy maximization when considering the model M2 (the ground truth in the case of unidirectional flow from $\mathrm{pop}_{j}$ to $\mathrm{pop}_{k}$ ) reflecting the good behaviour of the proposed method. 

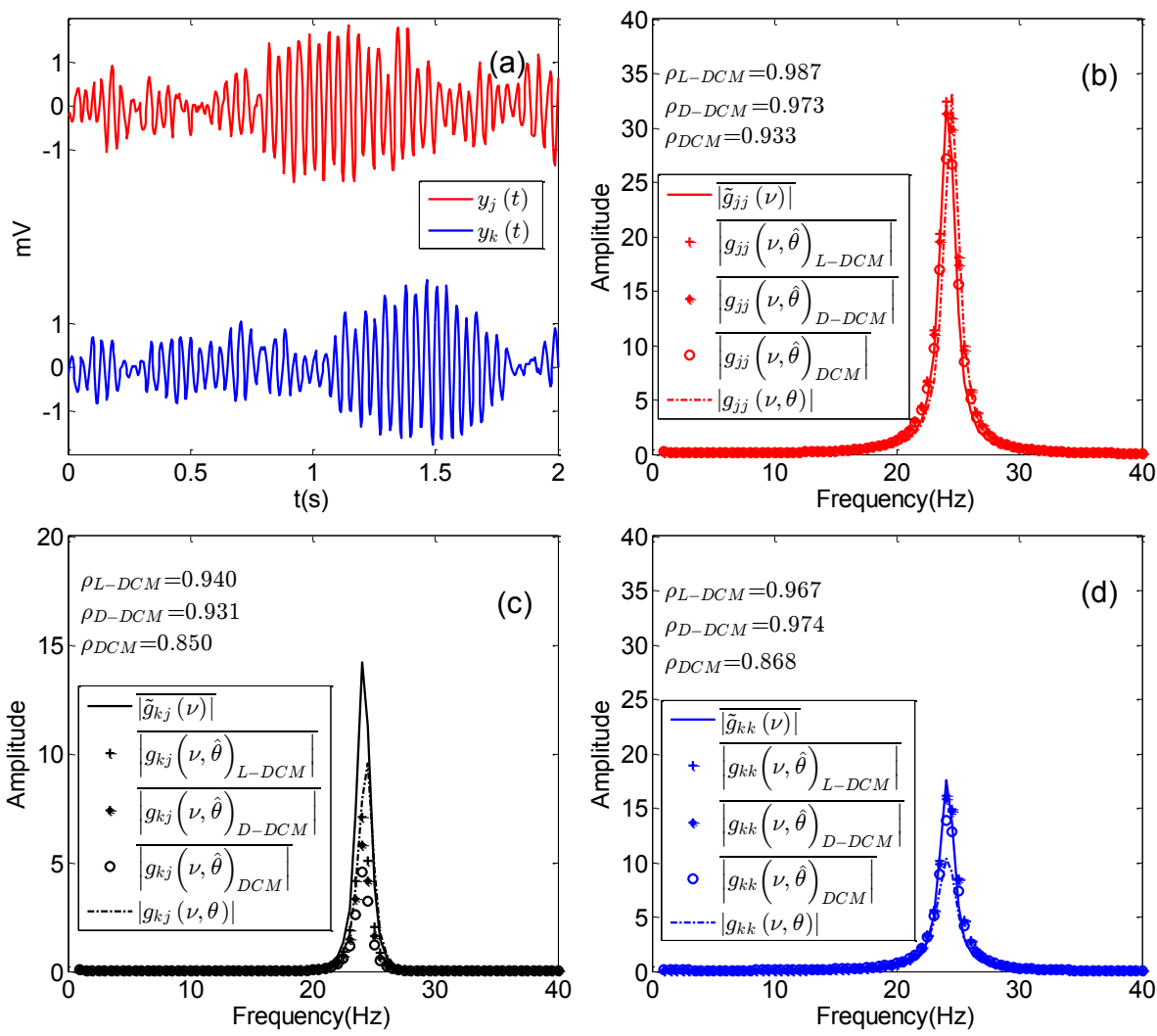

Fig. 3. Results corresponding to Scenario 3 when M2 is denoting the ground truth. (a) Simulated time signals displaying epileptic activities, (b), (c), (d) PSD magnitude (auto or cross PSD). The measured PSD is denoted by $\tilde{g}_{n}(\nu)$, $n \in\{j j, j k, k j, k k\}$, the estimated PSD using L-DCM $\left(\sigma^{2}=0.1\right)$, D-DCM and DCM by $g_{n}(\nu, \hat{\boldsymbol{\theta}})_{L-D C M}, g_{n}(\nu, \hat{\boldsymbol{\theta}})_{D-D C M}$ and $g_{n}(\nu, \hat{\boldsymbol{\theta}})_{D C M}$ respectively, and $g_{n}(\nu, \boldsymbol{\theta})$ is the analytical PSD using the true model parameters. $\rho_{L-D C M}$, $\rho_{D-D C M}$ and $\rho_{D C M}$ stand for the correlation coefficients between the measured and estimated PSD averaged over 100 trials for L-DCM $\left(\sigma^{2}=0.1\right)$, D-DCM and DCM approaches, respectively. 

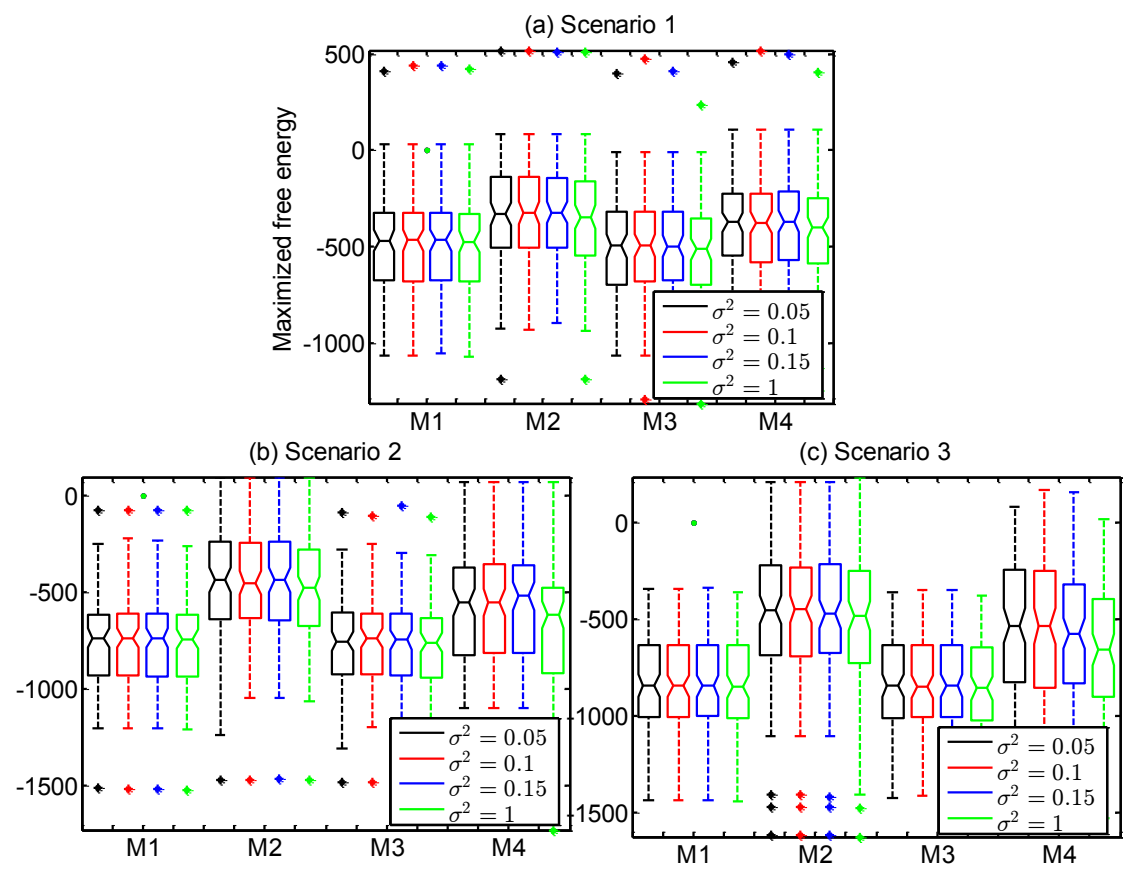

Fig. 4. Boxplots of the maximized free energy values using L-DCM with different values of $\sigma^{2}$ (unidirectional flow).

Besides, as expected, we note a lower free energy maximization when $\sigma^{2}=1$ compared to the other cases (i.e. $\sigma^{2}=0.05,0.1$ and 0.15 ). A slight superiority in maximizing the free energy for $\sigma^{2}=0.05$ and 0.1 compared to $\sigma^{2}=0.15$ is to be noticed whatever the scenario. Consequently, from now on, the variance $\sigma^{2}$ is set to 0.1 for the proposed L-DCM when compared to both the classical DCM [16] and the D-DCM [22] methods as shown hereafter.

\subsubsection{Performance analysis}

This subsection is devoted to evaluate the performance of the proposed L-DCM method in inferring neural structures underlying the simulated epileptic iEEG signals when unidirectional flow between neural assemblies is considered as prior (i.e. model M2 as ground truth). The behaviour of the proposed L-DCM method is compared to both the classical DCM [16] and the D-DCM [22] techniques. Table 3 shows both the model recognition rate and the execution time (in 
Table 3. Model selection over 100 trials using realistically simulated iEEG signals in the context of unidirectional flow

\begin{tabular}{|c|c|c|c|c|c|}
\hline \multirow[t]{2}{*}{ Scenario } & \multirow[t]{2}{*}{ Method } & \multicolumn{4}{|c|}{$\begin{array}{l}\text { Model identification count over } 100 \text { trials } \\
\text { (averaged run time over } 100 \text { trials (min)) }\end{array}$} \\
\hline & & M1 & M2 & M3 & M4 \\
\hline \multirow{3}{*}{$\left(K_{k j}=500\right)$} & L-DCM & $\begin{array}{l}6 / 100 \\
(12.2)\end{array}$ & $\begin{array}{c}\mathbf{8 1 / 1 0 0} \\
(26.7)\end{array}$ & $\begin{array}{l}5 / 100 \\
(24.0)\end{array}$ & $\begin{array}{l}8 / 100 \\
(31.3)\end{array}$ \\
\hline & D-DCM & $\begin{array}{c}12 / 100 \\
(4.7)\end{array}$ & $\begin{array}{c}\mathbf{7 9 / 1 0 0} \\
(9.0)\end{array}$ & $\begin{array}{l}3 / 100 \\
(11.2)\end{array}$ & $\begin{array}{l}6 / 100 \\
(17.1)\end{array}$ \\
\hline & DCM & $\begin{array}{c}13 / 100 \\
(2.1)\end{array}$ & $\begin{array}{c}73 / 100 \\
(4.8)\end{array}$ & $\begin{array}{c}4 / 100 \\
(5.6)\end{array}$ & $\begin{array}{c}10 / 100 \\
(8.9)\end{array}$ \\
\hline \multirow{3}{*}{$\left(K_{k j} \stackrel{2}{=} 1000\right)$} & L-DCM & $\begin{array}{l}0 / 100 \\
(13.2)\end{array}$ & $\begin{array}{c}\mathbf{8 4 / 1 0 0} \\
(38.5)\end{array}$ & $\begin{array}{l}1 / 100 \\
(24.1)\end{array}$ & $\begin{array}{c}15 / 100 \\
(42.7)\end{array}$ \\
\hline & D-DCM & $\begin{array}{c}0 / 100 \\
(4.2)\end{array}$ & $\begin{array}{c}\mathbf{9 6 / 1 0 0} \\
(12.5)\end{array}$ & $\begin{array}{l}1 / 100 \\
(10.2)\end{array}$ & $\begin{array}{l}3 / 100 \\
(21.8)\end{array}$ \\
\hline & DCM & $\begin{array}{c}1 / 100 \\
(2.0)\end{array}$ & $\begin{array}{c}\mathbf{9 6 / 1 0 0} \\
(6.0)\end{array}$ & $\begin{array}{c}1 / 100 \\
(5.1)\end{array}$ & $\begin{array}{c}2 / 100 \\
(9.8)\end{array}$ \\
\hline \multirow{3}{*}{$\left(K_{k j}=1500\right)$} & L-DCM & $\begin{array}{l}0 / 100 \\
(13.0)\end{array}$ & $\begin{array}{c}\mathbf{8 4 / 1 0 0} \\
(39.5)\end{array}$ & $\begin{array}{l}0 / 100 \\
(25.3)\end{array}$ & $\begin{array}{c}16 / 100 \\
(46.6)\end{array}$ \\
\hline & D-DCM & $\begin{array}{c}2 / 100 \\
(3.7)\end{array}$ & $\begin{array}{c}\mathbf{9 7 / 1 0 0} \\
(13.0)\end{array}$ & $\begin{array}{l}1 / 100 \\
(10.1)\end{array}$ & $\begin{array}{l}0 / 100 \\
(20.0)\end{array}$ \\
\hline & DCM & $\begin{array}{c}3 / 100 \\
(1.9)\end{array}$ & $\begin{array}{c}\mathbf{9 4 / 1 0 0} \\
(7.7)\end{array}$ & $\begin{array}{c}0 / 100 \\
(4.7)\end{array}$ & $\begin{array}{l}3 / 100 \\
(10.8)\end{array}$ \\
\hline
\end{tabular}

\section{(a) Scenario 1}

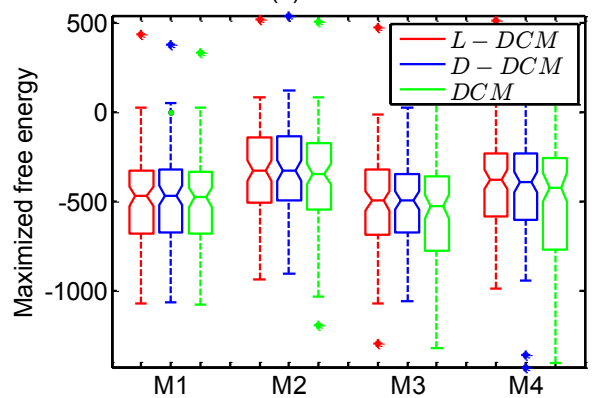

(b) Scenario 2

(c) Scenario 3
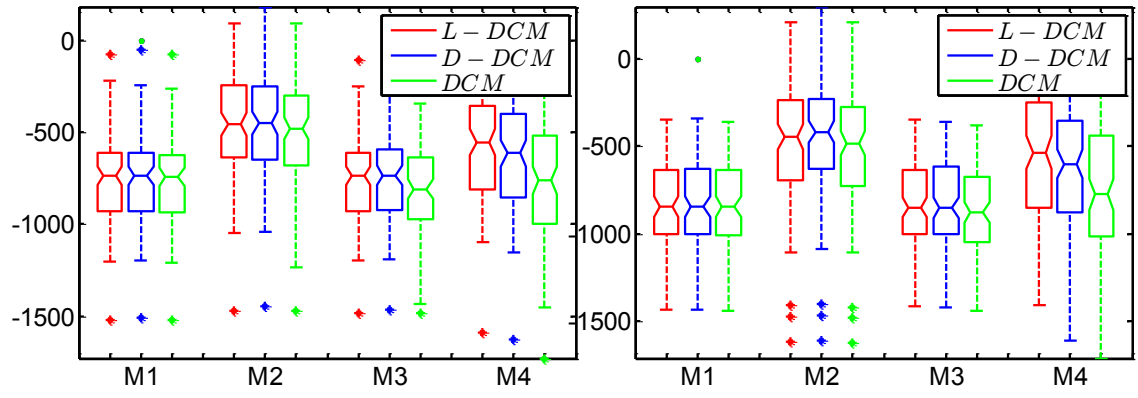

Fig. 5. Boxplots of the maximized free energy values using L-DCM $\left(\sigma^{2}=0.1\right)$, D-DCM and DCM for the 4 models (unidirectional flow). 
minutes) over 100 Monte Carlo trials. According to this table, generally, all techniques succeed in identifying the good model (model M2). However, the LDCM approach outperforms the two others (D-DCM and DCM) for low coupling strength ( $81 \%$ recognition rate vs. $79 \%$ and $73 \%$ respectively), whereas D-DCM outperforms DCM and L-DCM when $K_{k j}$ is equal to 1000 and 1500 (Scenario 2: 96\% recognition rate vs. $96 \%$ and $84 \%$ respectively, Scenario 3: $97 \%$ recognition rate vs. $94 \%$ and $84 \%$ respectively). In addition, Table 3 shows an increasing recognition rate for all methods in identifying the good model, M2, as $K_{k j}$ increases.

These results are further interpretable in terms of the maximized free energy as depicted in Fig. 5. Obviously, the maximal median value of the maximized free energy is globally obtained for model M2 whatever the method. In addition, both the L-DCM and D-DCM methods show relatively higher maximization of the free energy than the DCM one whatever the scenario and the model. Furthermore, some false identification situations (i.e. identifying either M1, M3 or M4 instead of the considered ground truth model M2) are noted in Table 3. Regarding the case of identifying M3 instead of M2, the false detection rate decreases from $5 \%$ to $0 \%$ for L-DCM, from $3 \%$ to $1 \%$ for D-DCM and from $4 \%$ to $0 \%$ for DCM when the coupling strength between $p o p_{j}$ and $p o p_{k}$ increases (i.e. from 500 to 1500). The same result holds for the case of identifying M1 instead of M2. As soon as M4 is concerned, Table 3 shows globally a higher false detection rate especially for L-DCM compared to the case of identifying M1 or M3 instead of M2. This issue could be explained by the fact that in case when the estimated coupling parameter $K_{j k}\left(\right.$ op $_{k} \rightarrow$ pop $\left._{j}\right)$ satisfies $K_{j k} \neq 0$ as it should not be since M2 is the target model, M4 can be possibly retained instead of M2. Indeed, a non-zero value of $K_{j k}$ leads in some way to a confusing choice between M2 and M4 which is not the case neither for M1 nor for M3. On the other hand, the Q1 Q3 quartiles of M4 are generally greater for L-DCM than for D-DCM and DCM as depicted in Fig. 5. Finally, we obtain a higher estimation quality of the L-DCM and D-DCM techniques over DCM in terms of PSD as clearly reflected by the values of the correlation coefficients, $\rho_{L-D C M}$ and $\rho_{D-D C M}$ vs $\rho_{D C M}$, 
computed between the estimated and measured PSDs, and depicted in Fig. 3 (b-d). These correlation coefficients are systematically higher for L-DCM or D-DCM than for DCM. As far as the execution time is concerned, Table 3 shows a higher execution time of L-DCM and D-DCM compared to DCM. This matter is clearly due to the iterative character of L-DCM when employing sweeps and D-DCM when using the DA scheme 22. However, this computation time is not crippling since higher PSD estimation and better free energy maximization are met with L-DCM and D-DCM compared to DCM.

\subsubsection{Bidirectional flow}

In this section, the bidirectional flow between $p o p_{j}$ and $p o p_{k}$ is considered so that M4 stands for the ground truth model to be inferred. This study is conducted as a function of the values of the couple $\left\{K_{k j}, K_{j k}\right\}$ reflecting the coupling strength between the two aforementioned epileptic populations. Three different couples of parameters $\left\{K_{k j}, K_{j k}\right\}$ are considered in this experiment leading to the three following experimental scenarios:

Scenario 1: $\left[A_{j} A_{k} B_{j} B_{k} G_{j} G_{k} K_{k j} K_{j k} \alpha_{j} \alpha_{k}\right]=[442225.525 .550050011]$

Scenario 2: $\left[A_{j} A_{k} B_{j} B_{k} G_{j} G_{k} K_{k j} K_{j k} \alpha_{j} \alpha_{k}\right]=[442235.535 .51000100011]$

Scenario 3: $\left[A_{j} A_{k} B_{j} B_{k} G_{j} G_{k} K_{k j} K_{j k} \alpha_{j} \alpha_{k}\right]=[442246.346 .31500150011]$

Only the case $K_{k j}=K_{j k}$ is considered in this study since only the symmetric influence of the coupling strength is tested. Note also that the above sets of parameters allow to produce effectively fast sinusoidal activity around $25 \mathrm{~Hz}$ similar to the epileptic one observed at the seizure onset (see Fig. 6 (a)).

\subsubsection{Choice of $\sigma^{2}$}

Similarly to the unidirectional flow case, the influence of the parameter $\sigma^{2}$ in the bidirectional flow on the maximization of the free energy in the L-DCM method was to be investigated. Therefore, six values of $\sigma^{2}$ were evaluated in this study, $\sigma^{2} \in\{0.05,0.1,0.15,0.2,0.8,1\}$, where 100 Monte Carlo trials were conducted for each value. Fig. 7 shows the boxplots of the maximized free energy corresponding to the bidirectional flow where results for $\sigma^{2}=0.2$ and 0.8 were 



Fig. 6. Results corresponding to Scenario 3 when M4 is denoting the ground truth. (a) Simulated time signals displaying epileptic activities, (b), (c), (d) PSD magnitude (auto or cross PSD). The measured PSD is denoted by $\tilde{g}_{n}(\nu)$, $n \in\{j j, j k, k j, k k\}$, the estimated PSD using L-DCM $\left(\sigma^{2}=0.1\right)$, D-DCM and DCM by $g_{n}(\nu, \hat{\boldsymbol{\theta}})_{L-D C M}, g_{n}(\nu, \hat{\boldsymbol{\theta}})_{D-D C M}$ and $g_{n}(\nu, \hat{\boldsymbol{\theta}})_{D C M}$ respectively, and $g_{n}(\nu, \boldsymbol{\theta})$ is the analytical PSD using the true model parameters. $\rho_{L-D C M}$, $\rho_{D-D C M}$ and $\rho_{D C M}$ stand for the correlation coefficients between the measured and estimated PSD averaged over 100 trials for L-DCM $\left(\sigma^{2}=0.1\right)$, D-DCM and DCM approaches, respectively. 


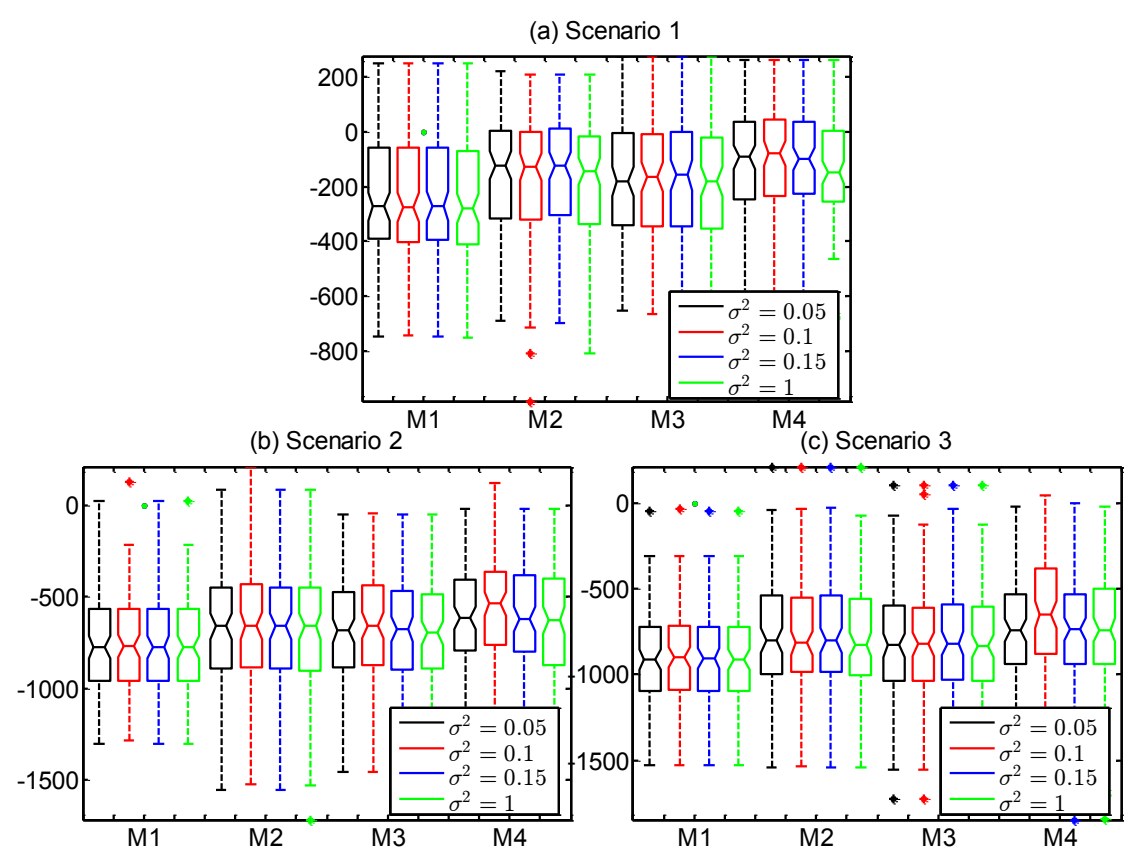

Fig. 7. Boxplots of the maximized free energy values using L-DCM and different values of $\sigma^{2}$ (bidirectional flow).

omitted since the results of the former were similar as the ones for $\sigma^{2}=0.15$ and the results of the latter were similar for the ones of $\sigma^{2}=1$. Note that the model M4 is correctly identified for all values of $\sigma^{2}$ as shown in Fig. 7 (a), (b), (c). We note from these figures that, regardless of the considered scenario, a higher free energy is generally obtained when $\sigma^{2}=0.1$. Also, a lower maximized free energy is obtained for $\sigma^{2}=1$ regardless of the scenario. This is probably due to the initialization inconsistency between two successive sweeps caused by adding a random vector with relatively high variance (see (11)). Based on the above results, we fixed $\sigma^{2}$ to 0.1 in the following analysis.

\subsubsection{Performance analysis}

In this section, we present a comparative performance study in the proposed L-DCM method, conventional DCM [16] and D-DCM 22] techniques in inferring neural structures underlying the simulated epileptic iEEG signals in the case 
Table 4. Model selection over 100 trials using realistically simulated iEEG signals in the context of bidirectional flow

\begin{tabular}{|c|c|c|c|c|c|}
\hline \multirow[t]{2}{*}{ Scenario } & \multirow[t]{2}{*}{ Method } & \multicolumn{4}{|c|}{$\begin{array}{l}\text { Model identification count over } 100 \text { trials } \\
\text { (averaged run time over } 100 \text { trials (min)) }\end{array}$} \\
\hline & & M1 & M2 & M3 & M4 \\
\hline \multirow{3}{*}{$\begin{array}{c}1 \\
\left(K_{k j}=K_{j k}=500\right)\end{array}$} & L-DCM & $\begin{array}{c}7 / 100 \\
(6.9)\end{array}$ & $\begin{array}{c}26 / 100 \\
(14.5)\end{array}$ & $\begin{array}{c}22 / 100 \\
(15.3)\end{array}$ & $\begin{array}{c}\mathbf{4 5 / 1 0 0} \\
(17.6)\end{array}$ \\
\hline & D-DCM & $\begin{array}{c}10 / 100 \\
(3.1)\end{array}$ & $\mathbf{3 9 / 1 0 0}$ & $\begin{array}{c}31 / 100 \\
(5.4)\end{array}$ & $\begin{array}{c}20 / 100 \\
(6.4)\end{array}$ \\
\hline & DCM & $\begin{array}{c}10 / 100 \\
(1.4)\end{array}$ & $\begin{array}{c}\mathbf{3 6} / \mathbf{1 0 0} \\
(3.5)\end{array}$ & $\begin{array}{c}26 / 100 \\
(5.3)\end{array}$ & $\begin{array}{c}28 / 100 \\
(4.2)\end{array}$ \\
\hline \multirow{3}{*}{$\stackrel{2}{\left(K_{k j}=K_{j k}=1000\right)}$} & L-DCM & $\begin{array}{l}2 / 100 \\
(10.7)\end{array}$ & $\begin{array}{c}19 / 100 \\
(23.2)\end{array}$ & $\begin{array}{c}23 / 100 \\
(23.8)\end{array}$ & $\begin{array}{c}\mathbf{5 6 / 1 0 0} \\
(25.4)\end{array}$ \\
\hline & D-DCM & $\begin{array}{c}6 / 100 \\
(3.3)\end{array}$ & $\begin{array}{c}35 / 100 \\
(5.9)\end{array}$ & $\mathbf{3 8 / 1 0 0}$ & $21 / 100$ \\
\hline & DCM & $\begin{array}{c}6 / 100 \\
(1.5)\end{array}$ & $\begin{array}{c}31 / 100 \\
(3.4)\end{array}$ & $\begin{array}{c}\mathbf{3 3 / 1 0 0} \\
(3.9)\end{array}$ & $\begin{array}{c}30 / 100 \\
(5.4)\end{array}$ \\
\hline \multirow{3}{*}{$\begin{array}{c}3 \\
\left(K_{k j}=K_{j k}=1500\right)\end{array}$} & L-DCM & $\begin{array}{l}0 / 100 \\
(11.6)\end{array}$ & $\begin{array}{c}19 / 100 \\
(23.7)\end{array}$ & $\begin{array}{c}19 / 100 \\
(24)\end{array}$ & $\begin{array}{c}\mathbf{6 2 / 1 0 0} \\
(29.9)\end{array}$ \\
\hline & D-DCM & $\begin{array}{c}2 / 100 \\
(3.2)\end{array}$ & $\begin{array}{c}\mathbf{3 9} / \mathbf{1 0 0} \\
(5.9)\end{array}$ & $\begin{array}{c}32 / 100 \\
(6.1)\end{array}$ & $\begin{array}{c}27 / 100 \\
(10.7)\end{array}$ \\
\hline & DCM & $\begin{array}{c}6 / 100 \\
(1.4)\end{array}$ & $\begin{array}{c}\mathbf{3 7 / 1 0 0} \\
(3.3)\end{array}$ & $\begin{array}{c}24 / 100 \\
(3.2)\end{array}$ & $\begin{array}{c}33 / 100 \\
(6.5)\end{array}$ \\
\hline
\end{tabular}

(a) Scenario 1

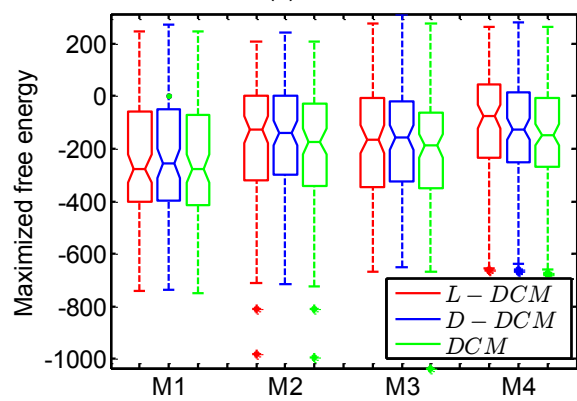

(b) Scenario 2

(c) Scenario 3



Fig. 8. Boxplots of the maximized free energy values using L-DCM $\left(\sigma^{2}=0.1\right)$, D-DCM and DCM for the 4 models (bidirectional flow). 
of bidirectional flow (i.e. model M4 as ground truth). Table 4 shows both the model recognition rate and the execution time (in minutes) over 100 Monte Carlo trials. According to this table, a highest recognition rate in identifying the good model (i.e. M4) is obtained by the proposed L-DCM method whatever the scenario. We note also that L-DCM outperforms both D-DCM and DCM in terms of the recognition rate regardless of the considered scenario. Besides, regarding the identification rate of $\mathrm{M} 4$, an improvement of $17 \%$ using L-DCM is noticed in Table 4 when the bidirectional coupling between the two populations increases. However, only an improvement of $7 \%$ and $5 \%$ is obtained using the D-DCM and DCM methods, respectively. Furthermore, despite the fact that all methods suffer from false identifications, the error rate of the proposed L-DCM method is still relatively smaller than the error rates of the D-DCM and DCM methods. Indeed, regarding the case of identifying M1 instead of M4, L-DCM shows systematically a lower false identification rate of $7 \%$ while $10 \%$ is observed using either D-DCM or DCM methods regardless of the considered scenario. Concerning the identification of models M2 and M3 instead of M4, the false detection rate using L-DCM is around $20 \%$ whereas it is more than $30 \%$ for both D-DCM and DCM.

Fig. 8 shows the boxplots of the maximized free energy using the three methods. According to this figure and regardless of the scenario, all techniques show higher median of the maximized free energy when identifying the model M4. More precisely, we note an improvement in maximizing the free energy using L-DCM and D-DCM compared to DCM, especially when identifying M4, but with some superiority for L-DCM. Consequently, the proposed L-DCM method leads to the best PSD estimation during the particular epileptic activity of interest. This claim is supported by considering the correlation coefficients, $\rho_{L-D C M}, \rho_{D-D C M}$ and $\rho_{D C M}$, computed between the PSDs of the observed and estimated epileptic signals as depicted in Fig. 6 (b-d). For instance, the correlation coefficient computed between the measured and estimated PSDs for the first population, is equal to 0.987 for L-DCM while it is equal to 0.986 for D-DCM and to 0.920 for DCM (see Fig. 6(b)). 


\subsection{Real $i E E G$ signals}

This subsection is devoted to evaluate the performance of both the proposed L-DCM method compared to the ones of the DCM and the D-DCM methods on real iEEG signals recorded on an epileptic patient. Our database is composed of 72-second length iEEG signals recorded using invasive electrodes equipped with 20 channels and placed in a specific region of the cerebral cortex. This specific brain structure is determined according to preliminary clinical and electrophysiological examinations. It is noteworthy that iEEG signals are all bipolar, i.e. obtained as the difference between the potentials recorded on two adjacent sensors. As shown in Fig. 9, each 72-second length signal, acquired with a $256 \mathrm{~Hz}$ sampling frequency, is divided into three phases: Pre-ictal $(0 \sim 20 \mathrm{~s})$, Ictal $(20 \sim 52 \mathrm{~s})$ and Post-ictal $(52 \sim 72 \mathrm{~s})$. According to the clinical expert, the Ictal phase $(20 \sim 52 \mathrm{~s})$ which corresponds to the epileptic seizure onset can be also divided into three overlapped epochs (named Ictal 1, Ictal 2 and Ictal 3 respectively). Both starting and ending points of each epoch are given in Table 5. According to the clinical expert, the 20 channels can be categorized into three groups according to their involvement along the different phases/epochs of the seizure. These groups are listed in Table 6 and named respectively Onset group (noted Group O), Propagation group (noted Group P) and Not-involved group (noted Group N). Let us indicate that signals associated with Group O are linked to activities in brain regions responsible for the seizure onset. Signals belonging to Group P are supposed to be influenced by those of the first group in the ictal phase. As for those included in Group N, they are assumed not to be impacted by the seizure.

\subsubsection{Analysis on a pair of channels}

As effective connectivity consists in inferring the directional flow between brain regions and since our purpose is to detect brain regions responsible for the seizure onset, it is legitimate to investigate this directionality by considering one channel extracted from Group $\mathrm{O}$ and another one issued from Group P. In this study, two channels are retained: the Pp4 channel, whose activity was 


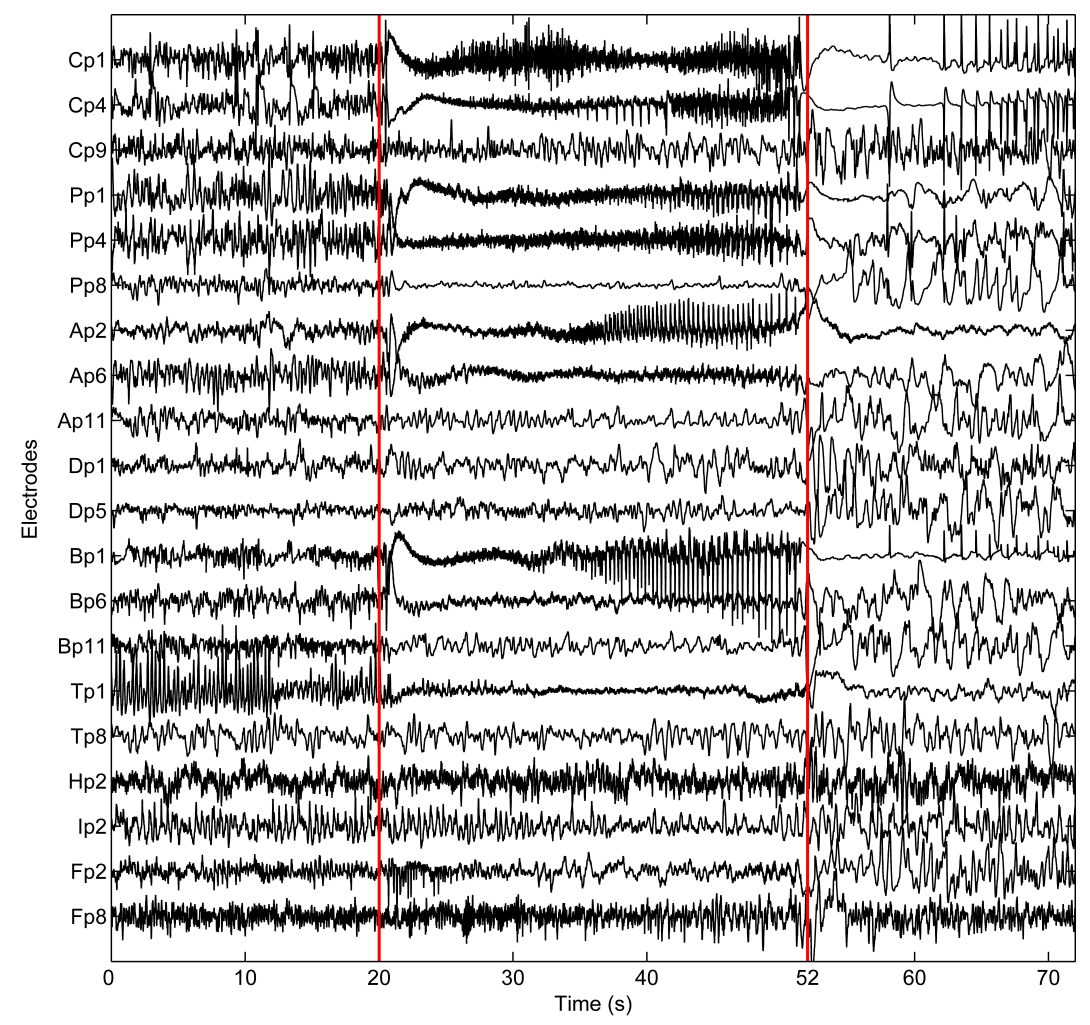

Fig. 9. A 72-second length iEEG recording with a seizure onset up to 32 seconds. Each channel corresponds to a bipolar iEEG signal. The two red vertical lines separate the recording into three segments: Pre-ictal phase $(0 \sim 20 \mathrm{~s})$, Ictal phase $(20 \sim 52 \mathrm{~s})$ and Post-ictal phase $(52 \sim 72 \mathrm{~s})$.

Table 5. Starting and Ending points of each epoch

\begin{tabular}{|c|c|}
\hline Epoch & Start point $\sim$ End point \\
\hline Pre-ictal & $2 \sim 18 \mathrm{~s}$ \\
\hline Ictal 1 & $22 \sim 38 \mathrm{~s}$ \\
\hline Ictal 2 & $28 \sim 46 \mathrm{~s}$ \\
\hline Ictal 3 & $34 \sim 50 \mathrm{~s}$ \\
\hline Post-ictal & $54 \sim 70 \mathrm{~s}$ \\
\hline
\end{tabular}


Table 6. Categories of the channels in the real iEEG for epileptic patient in the considered database

\begin{tabular}{ll}
\hline Group & Channels \\
\hline Onset group & $\mathrm{Cp} 1, \mathrm{Cp} 4, \mathrm{Pp} 1, \mathrm{Pp} 4, \mathrm{Ap} 2, \mathrm{Ap} 6, \mathrm{Bp} 1$ \\
Propagation group & $\mathrm{Pp} 8, \mathrm{Dp} 1, \mathrm{Tp} 1, \mathrm{Fp} 2$ \\
Not-involved group & $\mathrm{Cp} 9, \mathrm{Ap} 11, \mathrm{Dp} 5, \mathrm{Bp} 6, \mathrm{Bp} 11, \mathrm{Tp} 8, \mathrm{Hp} 2, \mathrm{Ip} 2, \mathrm{Fp} 8$ \\
\hline
\end{tabular}
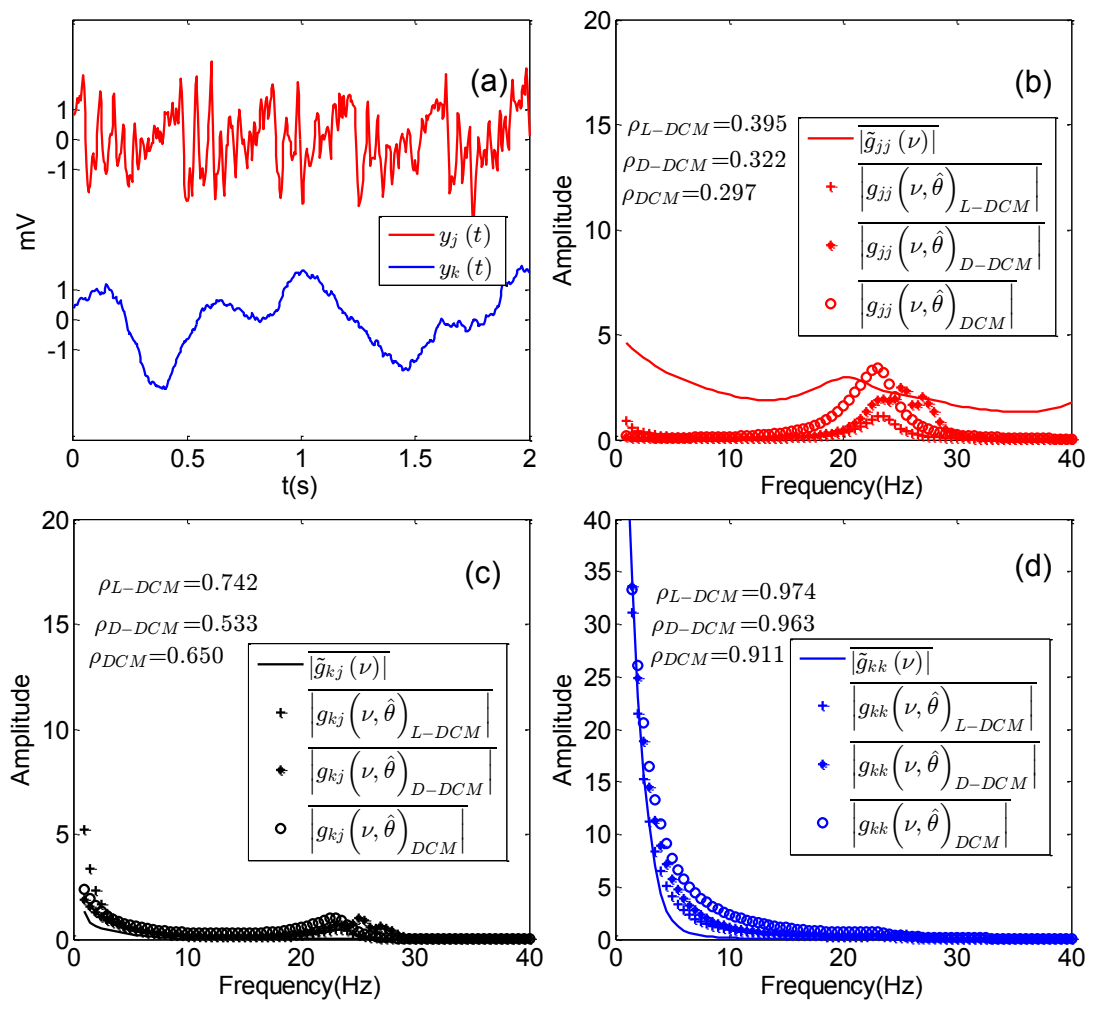

Fig. 10. Results corresponding to Scenario 3 when M2 is denoting the ground truth. (a) real iEEG signals displaying epileptic activities, (b), (c), (d) PSD magnitude (auto or cross PSD). The measured PSD is denoted by $\tilde{g}_{n}(\nu)$, $n \in\{j j, j k, k j, k k\}$, the estimated PSD using L-DCM $\left(\sigma^{2}=0.1\right)$, D-DCM and DCM by $g_{n}(\nu, \hat{\boldsymbol{\theta}})_{L-D C M}, g_{n}(\nu, \hat{\boldsymbol{\theta}})_{D-D C M}, g_{n}(\nu, \hat{\boldsymbol{\theta}})_{D C M}$ respectively. $\rho_{L-D C M}, \rho_{D-D C M}$ and $\rho_{D C M}$ stand for the correlation coefficients between the measured and estimated PSD averaged over 8 windows for L-DCM $\left(\sigma^{2}=0.1\right)$, D-DCM and DCM approaches, respectively. 
confirmed by the clinician to be part of the seizure onset zone (Group O) and the Dp1 channel which belongs to the Group P. It is worth mentioning that the bipolar signal derived from channel Pp4 (obtained as the difference between Pp4 and Pp5) is associated with the output $y_{j}(t)$ of population $p^{\circ} p_{j}$ whereas the bipolar signal derived from channel Dp1 (obtained as the difference between Dp1 and Dp2) is assigned with the output $y_{k}(t)$ of population pop $_{k}$. Effective connectivity between these two bipolar signals is evaluated for the three following epochs each of 16-second length giving rise to the three following scenarios: Scenario 1: Pre-ictal $(2 \sim 18 \mathrm{~s})$; Scenario 2: Ictal $1(22 \sim 38 \mathrm{~s})$ and Scenario 3: Ictal $2(30 \sim 46 \mathrm{~s})$. Each $16 \mathrm{~s}$ segment is partitioned using $2 \mathrm{~s}-\mathrm{sength}$ sliding windows without any overlap. For each scenario, each windowed signal segment of $2 \mathrm{~s}$ length is normalized and used to calculate the measured PSD, $\tilde{g}_{\ell \ell^{\prime}}(\nu), \ell, \ell^{\prime} \in\{j, k\}$. The recognition rate for all plausible models (i.e. M1, M2, M3 and M4) in the model space and the associated execution time (in minutes) are shown in Table 7 for the three considered approaches, namely the proposed L-DCM, the D-DCM and the DCM. A unidirectional connectivity from Pp4 to Dp1 (i.e. from Group O to Group P) for the three tested epochs (Pre-ictal, Ictal 1 and Ictal 2) is considered as a ground truth according to the clinician. Therefore, the model M2 stands for the ground truth structure for the three tested epochs and more particularly for the two ictal ones. According to Table 7. the proposed L-DCM method properly detects the propagation flow for these two temporal epochs. This claim is supported by the results depicted in Fig. 11 where generally more evidence to consider M2 among the other predefined models in the model space is obtained using the three methods. Moreover, a higher model evidence in terms of maximized free energy (as well as Q1 and Q3 quartiles) is obtained using the proposed L-DCM method compared to the two other techniques during the Ictal phase. This higher model evidence provided by the L-DCM method is associated with a better estimation of the PSD of the recorded signals. This is highlighted by the values of the correlation coefficients, $\rho_{L-D C M}, \rho_{D-D C M}$ and $\rho_{D C M}$, computed between the measured and estimated PSDs which are higher for L-DCM than for D-DCM and DCM in Fig. 10 (b-d). 
Table 7. Model selection over 8 2s-length windows for epileptic iEEG signals

\begin{tabular}{|c|c|c|c|c|c|}
\hline \multirow[t]{2}{*}{ Scenario } & \multirow[t]{2}{*}{ Method } & \multicolumn{4}{|c|}{$\begin{array}{l}\text { Model identification count over } 8 \text { windows } \\
\text { (averaged run time over } 8 \text { windows (min)) }\end{array}$} \\
\hline & & M1 & M2 & M3 & M4 \\
\hline \multirow{3}{*}{$\begin{array}{c}1 \\
(\text { Pre-ictal) }\end{array}$} & L-DCM & $\begin{array}{c}0 / 8 \\
(10.9)\end{array}$ & $\begin{array}{l}\mathbf{5} / \mathbf{8} \\
(8.2)\end{array}$ & $\begin{array}{c}2 / 8 \\
(13.4)\end{array}$ & $\begin{array}{l}1 / 8 \\
(8.5)\end{array}$ \\
\hline & D-DCM & $\begin{array}{c}2 / 8 \\
(17.6)\end{array}$ & $\begin{array}{c}2 / 8 \\
(12.7)\end{array}$ & $\begin{array}{c}2 / 8 \\
(12.3)\end{array}$ & $\begin{array}{c}2 / 8 \\
(27.4)\end{array}$ \\
\hline & DCM & $\begin{array}{c}0 / 8 \\
(6.0)\end{array}$ & $\begin{array}{l}\mathbf{6} / \mathbf{8} \\
(4.3)\end{array}$ & $\begin{array}{l}2 / 8 \\
(4.1)\end{array}$ & $\begin{array}{c}0 / 8 \\
(7.6)\end{array}$ \\
\hline \multirow{3}{*}{$\begin{array}{c}2 \\
(\text { Ictal } 1)\end{array}$} & L-DCM & $\begin{array}{c}0 / 8 \\
(18.8)\end{array}$ & $\begin{array}{l}\mathbf{8} / \mathbf{8} \\
(28.1)\end{array}$ & $\begin{array}{c}0 / 8 \\
(29.4)\end{array}$ & $\begin{array}{c}0 / 8 \\
(37.0)\end{array}$ \\
\hline & D-DCM & $\begin{array}{c}0 / 8 \\
(8.6)\end{array}$ & $\begin{array}{c}\mathbf{8} / \mathbf{8} \\
(17.9)\end{array}$ & $\begin{array}{c}0 / 8 \\
(17.2)\end{array}$ & $\begin{array}{c}0 / 8 \\
(9.2)\end{array}$ \\
\hline & DCM & $\begin{array}{c}0 / 8 \\
(5.3)\end{array}$ & $\begin{array}{l}\mathbf{5} / \mathbf{8} \\
(4.4)\end{array}$ & $\begin{array}{l}1 / 8 \\
(4.7)\end{array}$ & $\begin{array}{c}2 / 8 \\
(4.7)\end{array}$ \\
\hline \multirow{3}{*}{$\begin{array}{c}3 \\
\text { (Ictal 2) }\end{array}$} & L-DCM & $\begin{array}{c}0 / 8 \\
(22.1)\end{array}$ & $\begin{array}{c}\mathbf{8 / 8} \\
(27.0)\end{array}$ & $\begin{array}{c}0 / 8 \\
(31.3)\end{array}$ & $\begin{array}{c}0 / 8 \\
(36.9)\end{array}$ \\
\hline & D-DCM & $\begin{array}{c}0 / 8 \\
(11.9)\end{array}$ & $\begin{array}{c}\mathbf{5 / 8} \\
(23.0)\end{array}$ & $\begin{array}{c}0 / 8 \\
(30.6)\end{array}$ & $\begin{array}{c}0 / 8 \\
(32.2)\end{array}$ \\
\hline & DCM & $\begin{array}{c}0 / 8 \\
(5.0)\end{array}$ & $\begin{array}{c}\mathbf{7 / 8} \\
(5.1)\end{array}$ & $\begin{array}{c}1 / 8 \\
(5.0)\end{array}$ & $\begin{array}{c}0 / 8 \\
(5.4)\end{array}$ \\
\hline
\end{tabular}

(a) Scenario 1


Fig. 11. Boxplots of the maximized free energy values using L-DCM $\left(\sigma^{2}=0.1\right)$, D-DCM and DCM for the 4 models (real iEEG signals). 


\subsubsection{Group analysis}

The group analysis consists in investigating the information flow from Group O to Group P in the Pre-ictal and Ictal phases. As mentioned before, according to the clinician feedback, a unidirectional connection from Group O to Group P in Ictal phase, and to a lesser extent in Pre-ictal phase, could be considered as ground truth. Therefore, in both phases, but more surely in the Ictal phase, model M2 stands for the target model to infer. Following our previous study (section 4.2.1.), effective connectivity is quantified here for all possible unidirectional connections between channels belonging to Group $\mathrm{O}$ and channels belonging to Group P. Besides, for each considered method (i.e. L-DCM, D-DCM and $\mathrm{DCM}$ ), each channel in Group $\mathrm{O}$ is assimilated to the output $y_{j}(t)$ of population pop $_{j}$ whereas each channel in Group $\mathrm{P}$ is assimilated to the output $y_{k}(t)$ of

Table 8. Identified model structure and the corresponding median of the maximized free energy count over 8 2s-length windows using epileptic iEEG signals under Pre-ictal phase $(2 \sim 18 \mathrm{~s})$. C $C h 1 \in$ Group O and $C h 2 \in$ Group P

\begin{tabular}{|c|c|c|c|c|c|c|c|c|}
\hline \multirow{2}{*}{ Ch2 } & \multirow[t]{2}{*}{ Method } & \multicolumn{7}{|c|}{$\begin{array}{c}\text { Identified model structure } \\
\text { (Median of the maximized free energy) }\end{array}$} \\
\hline & & Cp1 & $\mathrm{Cp} 4$ & Pp1 & $\mathrm{Pp} 4$ & Ap2 & Ap6 & Bp1 \\
\hline \multirow{3}{*}{ Pp8 } & L-DCM & $\begin{array}{c}\mathrm{M} 2 \\
(\mathbf{- 9 4 8 )}\end{array}$ & \begin{tabular}{c|}
$\mathrm{M} 4$ \\
$(-1025)$
\end{tabular} & \begin{tabular}{c|}
$\mathrm{M} 2$ \\
$(-1272)$
\end{tabular} & $\begin{array}{c}\text { M4 } \\
(-1107)\end{array}$ & \begin{tabular}{c|}
$\mathrm{M} 4$ \\
$(-1091)$
\end{tabular} & $\begin{array}{c}\text { M1 } \\
(-1124)\end{array}$ & $\begin{array}{c}\mathrm{M} 4 \\
(-\mathbf{1 0 0 0})\end{array}$ \\
\hline & D-DCM & $\begin{array}{c}\text { M1 } \\
(-954)\end{array}$ & $\begin{array}{c}\mathrm{M} 4 \\
(\mathbf{- 1 0 2 0})\end{array}$ & $\begin{array}{c}\mathrm{M} 4 \\
\mathbf{( - 1 2 5 9 )}\end{array}$ & $\begin{array}{c}\mathrm{M} 2 \\
\mathbf{( - 1 0 9 4 )}\end{array}$ & $\begin{array}{c}\mathrm{M} 4 \\
(\mathbf{- 1 0 7 4})\end{array}$ & $\begin{array}{c}\text { M1 } \\
(\mathbf{- 1 1 2 0})\end{array}$ & $\begin{array}{c}\text { M1 } \\
(-1007)\end{array}$ \\
\hline & DCM & $\begin{array}{c}\text { M1 } \\
(-973)\end{array}$ & $\begin{array}{c}\mathrm{M} 2 \\
(-1106)\end{array}$ & $\begin{array}{c}\text { M1 } \\
(-1327)\end{array}$ & $\begin{array}{c}\text { M1 } \\
(-1137)\end{array}$ & $\begin{array}{c}\mathrm{M} 4 \\
(-1111)\end{array}$ & $\begin{array}{c}\text { M1 } \\
(-1139)\end{array}$ & $\begin{array}{c}\mathrm{M} 4 \\
(-1014)\end{array}$ \\
\hline \multirow{3}{*}{ Dp1 } & L-DCM & $\begin{array}{c}\mathrm{M} 2 \\
(\mathbf{- 9 6 0 )}\end{array}$ & \begin{tabular}{c|} 
M1 \\
$(-1132)$
\end{tabular} & $\begin{array}{c}\mathrm{M} 3 \\
(\mathbf{- 1 1 5 2})\end{array}$ & $\begin{array}{c}\mathrm{M} 2 \\
(-1152)\end{array}$ & $\begin{array}{c}\mathrm{M} 3 \\
(-\mathbf{1 1 1 7})\end{array}$ & $\begin{array}{c}\text { M1 } \\
(-1051)\end{array}$ & $\begin{array}{c}\mathrm{M} 4 \\
(-1018)\end{array}$ \\
\hline & D-DCM & $\begin{array}{c}\text { M2 } \\
(-973)\end{array}$ & $\begin{array}{c}\mathrm{M} 4 \\
(\mathbf{- 1 1 1 7})\end{array}$ & $\begin{array}{c}\text { M1 } \\
(-1154)\end{array}$ & $\begin{array}{c}\mathrm{M} 4 \\
(\mathbf{- 1 0 9 0})\end{array}$ & $\begin{array}{c}\mathrm{M} 2 \\
(-1219)\end{array}$ & $\begin{array}{c}M 1 \\
(\mathbf{- 1 0 5 0})\end{array}$ & $\begin{array}{c}\mathrm{M} 4 \\
\mathbf{( - 1 0 1 2 )}\end{array}$ \\
\hline & DCM & $\begin{array}{c}\text { M2 } \\
(-1022) \\
\end{array}$ & $\begin{array}{c}\text { M1 } \\
(-1146) \\
\end{array}$ & $\begin{array}{c}\text { M1 } \\
(-1172) \\
\end{array}$ & $\begin{array}{c}\text { M2 } \\
(-1154)\end{array}$ & $\begin{array}{c}\text { M2 } \\
(-1263) \\
\end{array}$ & $\begin{array}{c}\text { M4 } \\
(-1077)\end{array}$ & $\begin{array}{c}\text { M4 } \\
(-1039) \\
\end{array}$ \\
\hline \multirow{3}{*}{ Tp1 } & L-DCM & $\begin{array}{c}\text { M4 } \\
(-949)\end{array}$ & $\begin{array}{c}\text { M4 } \\
(-1077)\end{array}$ & $\begin{array}{c}\text { M4 } \\
(-1175)\end{array}$ & $\begin{array}{c}\mathrm{M} 4 \\
(\mathbf{- 1 0 3 6})\end{array}$ & $\begin{array}{c}\mathrm{M} 4 \\
(-\mathbf{1 0 6 7})\end{array}$ & $\begin{array}{c}\mathrm{M} 4 \\
(\mathbf{- 1 1 4 3})\end{array}$ & $\begin{array}{c}\text { M3 } \\
(-993)\end{array}$ \\
\hline & D-DCM & $\begin{array}{c}\text { M2 } \\
(\mathbf{- 9 3 7 )}\end{array}$ & $\begin{array}{c}\mathrm{M} 2 \\
\mathbf{( - 1 0 6 8 )}\end{array}$ & $\begin{array}{c}\mathrm{M} 3 \\
(\mathbf{- 1 1 7 0})\end{array}$ & $\begin{array}{c}\mathrm{M} 4 \\
(-1043)\end{array}$ & $\begin{array}{c}\mathrm{M} 4 \\
(-1087)\end{array}$ & $\begin{array}{c}\mathrm{M} 2 \\
(-1151)\end{array}$ & $\begin{array}{c}\text { M2 } \\
(\mathbf{- 9 3 8})\end{array}$ \\
\hline & DCM & $\begin{array}{c}\text { M4 } \\
(-966)\end{array}$ & $\begin{array}{c}\mathrm{M} 4 \\
(-1092)\end{array}$ & $\begin{array}{c}\mathrm{M} 4 \\
(-1216)\end{array}$ & $\begin{array}{c}\text { M4 } \\
(-1051)\end{array}$ & $\begin{array}{c}\text { M3 } \\
(-1095)\end{array}$ & $\begin{array}{c}\mathrm{M} 2 \\
(-1185)\end{array}$ & $\begin{array}{c}\text { M3 } \\
(-1039)\end{array}$ \\
\hline \multirow{3}{*}{ Fp2 } & L-DCM & $\begin{array}{c}\text { M3 } \\
(\mathbf{- 8 0 8 )}\end{array}$ & $\begin{array}{c}\text { M3 } \\
(-\mathbf{9 8 3})\end{array}$ & $\begin{array}{c}\mathrm{M} 2 \\
(-1057)\end{array}$ & $\begin{array}{c}\text { M4 } \\
(-\mathbf{9 8 6})\end{array}$ & $\begin{array}{c}\text { M3 } \\
(-1039)\end{array}$ & $\begin{array}{c}\mathrm{M} 3 \\
\mathbf{( - 9 8 0 )}\end{array}$ & $\begin{array}{c}\text { M4 } \\
(-887)\end{array}$ \\
\hline & D-DCM & $\begin{array}{c}\text { M4 } \\
(-840)\end{array}$ & $\begin{array}{c}\text { M3 } \\
(-991)\end{array}$ & $\begin{array}{c}\mathrm{M} 2 \\
(\mathbf{- 1 0 5 3})\end{array}$ & $\begin{array}{c}\text { M4 } \\
(-992)\end{array}$ & $\begin{array}{c}\mathrm{M} 2 \\
(\mathbf{- 9 1 4 )}\end{array}$ & $\begin{array}{c}\text { M4 } \\
(-1023)\end{array}$ & $\begin{array}{c}\text { M2 } \\
(-856)\end{array}$ \\
\hline & DCM & $\begin{array}{c}\text { M4 } \\
(-863)\end{array}$ & $\begin{array}{c}\text { M3 } \\
(-992)\end{array}$ & $\begin{array}{c}\text { M4 } \\
(-1091)\end{array}$ & $\begin{array}{c}\text { M4 } \\
(-1003)\end{array}$ & $\begin{array}{c}\text { M1 } \\
(-1062)\end{array}$ & $\begin{array}{c}\text { M4 } \\
(-1073)\end{array}$ & $\begin{array}{c}\text { M4 } \\
(-915)\end{array}$ \\
\hline
\end{tabular}


Table 9. Identified model structure and the corresponding median of the maximized free energy count over 12 2s-length windows using epileptic iEEG signals under Ictal phase $(22 \sim 46 \mathrm{~s})$. $C h 1 \in$ Group $\mathrm{O}$ and $C h 2 \in$ Group P

\begin{tabular}{|c|c|c|c|c|c|c|c|c|}
\hline \multirow{2}{*}{$C h 2$} & \multirow[t]{2}{*}{ Method } & \multicolumn{7}{|c|}{$\begin{array}{c}\text { Identified model structure } \\
\text { (median of the maximized free energy) }\end{array}$} \\
\hline & & Cp1 & $\mathrm{Cp} 4$ & Pp1 & $\mathrm{Pp} 4$ & Ap2 & Ap6 & Bp1 \\
\hline \multirow{3}{*}{ Pp8 } & L-DCM & $\begin{array}{c}\mathrm{M} 2 \\
(\mathbf{- 5 8 6})\end{array}$ & $\begin{array}{c}\mathrm{M} 2 \\
(\mathbf{- 8 2 4})\end{array}$ & $\begin{array}{c}\mathrm{M} 2 \\
(\mathbf{- 8 8 6})\end{array}$ & $\begin{array}{c}\mathrm{M} 2 \\
(-\mathbf{7 1 4})\end{array}$ & $\begin{array}{c}\mathrm{M} 2 \\
(\mathbf{- 8 5 1})\end{array}$ & $\begin{array}{c}\mathrm{M} 2 \\
(\mathbf{- 1 0 5 1 )}\end{array}$ & $\begin{array}{c}\mathrm{M} 2 \\
(\mathbf{- 8 4 0})\end{array}$ \\
\hline & D-DCM & $\begin{array}{c}\mathrm{M} 2 \\
(-653)\end{array}$ & $\begin{array}{c}\mathrm{M} 2 \\
(-922)\end{array}$ & $\begin{array}{c}\text { M2 } \\
(-930)\end{array}$ & $\begin{array}{c}\text { M2 } \\
(-750)\end{array}$ & $\begin{array}{c}\mathrm{M} 2 \\
(-906)\end{array}$ & $\begin{array}{c}\text { M2 } \\
(-1068)\end{array}$ & $\begin{array}{c}\text { M2 } \\
(-936)\end{array}$ \\
\hline & DCM & $\begin{array}{c}\mathrm{M} 2 \\
(-751)\end{array}$ & $\begin{array}{c}\mathrm{M} 2 \\
(-937)\end{array}$ & $\begin{array}{c}\text { M2 } \\
(-971)\end{array}$ & $\begin{array}{c}\text { M2 } \\
(-788)\end{array}$ & $\begin{array}{c}\text { M2 } \\
(-997)\end{array}$ & $\begin{array}{c}\text { M2 } \\
(-1109)\end{array}$ & $\begin{array}{c}\text { M2 } \\
(-988)\end{array}$ \\
\hline \multirow{3}{*}{ Dp1 } & L-DCM & $\begin{array}{c}\text { M2 } \\
(-\mathbf{7 9 8})\end{array}$ & $\begin{array}{c}\mathrm{M} 2 \\
(\mathbf{- 8 6 6 )}\end{array}$ & $\begin{array}{c}\text { M2 } \\
(-\mathbf{7 1 9})\end{array}$ & $\begin{array}{c}\mathrm{M} 2 \\
(\mathbf{- 8 1 3})\end{array}$ & $\begin{array}{c}\text { M2 } \\
(-955)\end{array}$ & $\begin{array}{c}\mathrm{M} 2 \\
(\mathbf{- 1 0 3 0})\end{array}$ & $\begin{array}{c}\mathrm{M} 2 \\
\mathbf{( - 9 4 1 )}\end{array}$ \\
\hline & D-DCM & $\begin{array}{c}\text { M2 } \\
(-826)\end{array}$ & $\begin{array}{c}\text { M2 } \\
(-888)\end{array}$ & $\begin{array}{c}\text { M2 } \\
(-846)\end{array}$ & $\begin{array}{c}\text { M2 } \\
(-821)\end{array}$ & $\begin{array}{c}\text { M2 } \\
(\mathbf{- 9 2 5})\end{array}$ & $\begin{array}{c}\text { M2 } \\
(-1067)\end{array}$ & $\begin{array}{c}\text { M2 } \\
(-983)\end{array}$ \\
\hline & DCM & $\begin{array}{c}\text { M2 } \\
(-872)\end{array}$ & $\begin{array}{c}\text { M2 } \\
(-938)\end{array}$ & $\begin{array}{c}\text { M2 } \\
(-883)\end{array}$ & $\begin{array}{c}\text { M2 } \\
(-860)\end{array}$ & $\begin{array}{c}\text { M2 } \\
(-1012)\end{array}$ & $\begin{array}{c}\text { M2 } \\
(-1087)\end{array}$ & $\begin{array}{c}\text { M2 } \\
(-1005)\end{array}$ \\
\hline \multirow{3}{*}{ Tp1 } & L-DCM & $\begin{array}{c}\text { M2 } \\
(-496)\end{array}$ & $\begin{array}{c}\text { M4 } \\
(-786)\end{array}$ & $\begin{array}{c}\mathrm{M} 2 \\
(\mathbf{- 6 2 4})\end{array}$ & $\begin{array}{c}\text { M2 } \\
(\mathbf{- 5 8 9})\end{array}$ & $\begin{array}{c}\text { M3 } \\
(-782)\end{array}$ & $\begin{array}{c}\text { M2 } \\
(\mathbf{- 8 9 2 )}\end{array}$ & $\begin{array}{c}\text { M2 } \\
(-727)\end{array}$ \\
\hline & D-DCM & $\begin{array}{c}\text { M2 } \\
(-\mathbf{4 8 7})\end{array}$ & $\begin{array}{c}\mathrm{M} 2 \\
(\mathbf{- 6 8 8})\end{array}$ & $\begin{array}{c}\text { M2 } \\
(-638)\end{array}$ & $\begin{array}{c}\text { M2 } \\
(-610)\end{array}$ & $\begin{array}{c}\text { M4 } \\
(-807)\end{array}$ & $\begin{array}{c}\text { M2 } \\
(-945)\end{array}$ & $\begin{array}{c}\text { M2 } \\
(\mathbf{- 7 2 4})\end{array}$ \\
\hline & DCM & $\begin{array}{c}\text { M2 } \\
(-546)\end{array}$ & $\begin{array}{c}\text { M1 } \\
(-808)\end{array}$ & $\begin{array}{c}\text { M2 } \\
(-756)\end{array}$ & $\begin{array}{c}\text { M2 } \\
(-662)\end{array}$ & $\begin{array}{c}\text { M4 } \\
(-859)\end{array}$ & $\begin{array}{c}\text { M1 } \\
(-981)\end{array}$ & $\begin{array}{c}\text { M2 } \\
(-786)\end{array}$ \\
\hline \multirow{3}{*}{ Fp2 } & L-DCM & $\begin{array}{c}\mathrm{M} 2 \\
(\mathbf{- 5 2 3})\end{array}$ & $\begin{array}{c}\text { M2 } \\
(-814)\end{array}$ & $\begin{array}{c}\mathrm{M} 2 \\
(\mathbf{- 6 9 0})\end{array}$ & $\begin{array}{c}\mathrm{M} 2 \\
(\mathbf{- 6 2 2})\end{array}$ & $\begin{array}{c}\text { M2 } \\
(\mathbf{- 8 2 6 )}\end{array}$ & $\begin{array}{c}\text { M2 } \\
(\mathbf{- 9 9 8 )}\end{array}$ & $\begin{array}{c}\text { M2 } \\
(\mathbf{- 9 2 4 )}\end{array}$ \\
\hline & D-DCM & $\begin{array}{c}\mathrm{M} 2 \\
(-575)\end{array}$ & $\begin{array}{c}\mathrm{M} 2 \\
(\mathbf{- 8 0 5})\end{array}$ & $\begin{array}{c}\text { M2 } \\
(-762)\end{array}$ & $\begin{array}{c}\text { M2 } \\
(-642)\end{array}$ & $\begin{array}{c}\mathrm{M} 2 \\
(-894)\end{array}$ & $\begin{array}{c}\text { M2 } \\
(-1024)\end{array}$ & $\begin{array}{c}\text { M2 } \\
(-960)\end{array}$ \\
\hline & DCM & $\begin{array}{c}\text { M2 } \\
(-617)\end{array}$ & $\begin{array}{c}\text { M2 } \\
(-864)\end{array}$ & $\begin{array}{c}\text { M2 } \\
(-790)\end{array}$ & $\begin{array}{c}\text { M2 } \\
(-692)\end{array}$ & $\begin{array}{c}\text { M2 } \\
(-882)\end{array}$ & $\begin{array}{c}\text { M2 } \\
(-1063)\end{array}$ & $\begin{array}{c}\text { M4 } \\
(-978)\end{array}$ \\
\hline
\end{tabular}

population pop $_{k}$. Tables 8 and 9 display the identified model structure and the corresponding median of the maximized free energy for each couple of channels $(C h 1, C h 2)$ where $C h 1 \in$ Group $\mathrm{O}$ and $C h 2 \in$ Group P, respectively during the Pre-ictal phase (i.e. $(2 \sim 18 s))$ and the Ictal phase (i.e. $(22 \sim 46 s)$ ).

According to the results depicted in Table 8 a high variability in recognizing the true model (i.e. M2) whatever the $(C h 1, C h 2)$ couple and regardless of the considered method can be observed. Now, D-DCM and L-DCM present a comparable behaviour in terms of maximizing the free energy, this free energy being generally greater than using the conventional DCM method. The identification of Model 2 is not so easy: for instance, the L-DCM method correctly recognizes the good model for the couples $(\mathrm{Cp} 1, \mathrm{Pp} 8),(\mathrm{Cp} 1, \mathrm{Dp} 1)$ whereas the latter succeeds in identifying the true model for the couples (Cp1, Dp1) and 
(Cp1, Tp1). This high variability is also observed on other couples and can be explained by rapid changes in the interactions between different regions of the brain during the Pre-ictal phase wherein the seizure propagation starts to be set up.

Regarding the Ictal phase and the three methods (Table 9), it comes out that most of the channels in Group O have a causal influence on those in Group P. Therefore, the true model M2 is identified most of the time. In addition, the proposed L-DCM method generally provides higher median values of the maximized free energy compared to both D-DCM and DCM. For example, given the couple (Cp4, Pp8), L-DCM leads to a median value of the maximized free energy equal to -824 whereas D-DCM and DCM lead to values equal to -922 and -937 respectively.

To conclude on this analysis, our results globally match with the analysis of the clinician especially for the Ictal phase.

\section{Discussion}

In this study, to analyze human epileptic signals, three DCM based methods have been applied and compared. Tested on simulated epileptic iEEG data and real signals, the proposed L-DCM algorithm outperformed the two others in terms of maximizing the free energy. This is thanks to the use of well-conditioned variational EM sweeps during the maximization process. Besides, L-DCM shows particularly higher performance in identifying the bidirectional propagation flow. Regarding the unidirectional flow, all techniques displayed quite comparable results even if L-DCM outperformed the two other DCM approaches in case of low coupling strength between the epileptic populations. It is noteworthy that since the epileptic seizure is typically patient-dependent, investigating the performance of the proposed L-DCM method using other epileptic iEEG dataset would be interesting and will be our next objective. 


\section{Conclusion}

In this paper, we proposed a local adjustment model-based approach, named L-DCM, to quantify effective connectivity between different brain regions involved in the onset and in the propagation of epileptic seizures. The proposed method is robust to unsuitable initialization of the model parameters contrary to the standard DCM and D-DCM approaches. This is the result of using several wellconditioned variational EM sweeps during the maximization process. In addition, as mentioned previously, the proposed L-DCM method generally showed higher performance for both unidirectional and bidirectional flows especially for low coupling strength in the first case.

Coming works will consist in improving the accuracy of model identification. We plan to consider a new generative model like the modified physiology-based model given in 25]. Accordingly, the L-DCM will be adapted to take into account the intrinsic structure of this new model. These variants should be evaluated on an extended real epileptic iEEG dataset to take into account the variability of the epileptic seizure among patients.

\section{Acknowledgment}

This work was supported by the Scientific Emerging Challenges 12FA69101, the NSFC under Grant 31400842, Natural Science Foundation of Jiangsu Province under Grant DZXX-031, BY2014127-11 and the '333' project under Grant BRA2015288.

\section{References}

[1] F. Wendling, P. Chauvel, A. Biraben, F. Bartolomei, From intracerebral eeg signals to brain connectivity: identification of epileptogenic networks in partial epilepsy, Frontiers in systems neuroscience 4 (2010) 154.

[2] M. Rubinov, O. Sporns, Complex network measures of brain connectivity: uses and interpretations, Neuroimage 52 (3) (2010) 1059-1069. 
[3] N. Wiener, The theory of prediction, Modern mathematics for engineers 1 (1956) 125-139.

[4] C. W. Granger, Investigating causal relations by econometric models and cross-spectral methods, Econometrica: Journal of the Econometric Society (1969) 424-438.

[5] Y. Saito, H. Harashima, Tracking of information within multichannel EEG record causal analysis in EEG, Yamaguchi N, Fujisawa K (eds) Recent advances in EEG and EMG data processing. Elsevier (1981) 133-146.

[6] J. Geweke, Measurement of linear dependence and feedback between multiple time series, Journal of the American statistical association 77 (378) (1982) 304-313.

[7] K. J. Blinowska, R. Kuś, M. Kamiński, Granger causality and information flow in multivariate processes, Physical Review E 70 (5) (2004) 050902.

[8] T. Schreiber, Measuring information transfer, Physical review letters 85 (2) (2000) 461.

[9] A. Razi, K. J. Friston, The connected brain: Causality, models, and intrinsic dynamics, IEEE Signal Processing Magazine 33 (3) (2016) 14-35.

[10] K. J. Friston, L. Harrison, W. Penny, Dynamic causal modelling, NeuroImage 19 (4) (2003) 1273-1302.

[11] J. Daunizeau, K. E. Stephan, K. J. Friston, Stochastic dynamic causal modelling of fmri data: should we care about neural noise?, Neuroimage 62 (1) (2012) 464-81.

[12] R. Moran, D. A. Pinotsis, K. Friston, Neural masses and fields in dynamic causal modeling, Neural Masses and Fields: Modelling the Dynamics of Brain Activity (2015) 190. 
[13] K. J. Friston, A. Bastos, V. Litvak, K. E. Stephan, P. Fries, R. J. Moran, Dcm for complex-valued data: cross-spectra, coherence and phase-delays, Neuroimage 59 (1) (2012) 439-55.

[14] R. J. Moran, S. J. Kiebel, K. E. Stephan, R. B. Reilly, J. Daunizeau, K. J. Friston, A neural mass model of spectral responses in electrophysiology, Neuroimage 37 (3) (2007) 706-20.

[15] R. J. Moran, K. E. Stephan, T. Seidenbecher, H. C. Pape, R. J. Dolan, K. J. Friston, Dynamic causal models of steady-state responses, Neuroimage 44 (3) (2009) 796-811.

[16] W. Xiang, C. Yang, J.-J. Bellanger, H. Shu, R. L. B. Jeannès, Inferring effective connectivity in epilepsy using dynamic causal modeling, IRBM 36 (6) (2015) 335-344.

[17] M. Papadopoulou, M. Leite, P. van Mierlo, K. Vonck, L. Lemieux, K. J. Friston, D. Marinazzo, Tracking slow modulations in synaptic gain using dynamic causal modelling: validation in epilepsy, NeuroImage 107 (2015) $117-126$.

[18] G. K. Cooray, B. Sengupta, P. K. Douglas, K. J. Friston, Dynamic causal modelling of electrographic seizure activity using bayesian belief updating, Neuroimage 125 (2016) 1142-1154.

[19] J. Daunizeau, K. J. Friston, S. J. Kiebel, Variational bayesian identification and prediction of stochastic nonlinear dynamic causal models, Physica D 238 (21) (2009) 2089-2118.

[20] K. Friston, J. Mattout, N. Trujillo-Barreto, J. Ashburner, W. Penny, Variational free energy and the laplace approximation, Neuroimage 34 (1) (2007) 220-34.

[21] N. Ueda, R. Nakano, Deterministic annealing em algorithm, Neural Networks 11 (2) (1998) 271-282. 
[22] K. Katahira, K. Watanabe, M. Okada, Deterministic annealing variant of variational bayes method 95 (1) (2008) 012015.

[23] B. Sengupta, K. J. Friston, W. D. Penny, Gradient-based mcmc samplers for dynamic causal modelling, NeuroImage 125 (2016) 1107-1118.

[24] F. Wendling, A. Hernandez, J.-J. Bellanger, P. Chauvel, F. Bartolomei, Interictal to ictal transition in human temporal lobe epilepsy: insights from a computational model of intracerebral eeg, Journal of Clinical Neurophysiology 22 (5) (2005) 343.

[25] M. Ursino, F. Cona, M. Zavaglia, The generation of rhythms within a cortical region: analysis of a neural mass model, NeuroImage 52 (3) (2010) 1080-1094. 